BUTP-94/12

HUTP-94/A012

\title{
Effective Field Theory of the Linear $O(N)$ Sigma Model $q$
}

\author{
A. Nyffeler \\ Institute for Theoretical Physics \\ University of Bern \\ Sidlerstrasse 5, CH-3012 Bern, Switzerland \\ and \\ A. Schenk \\ The Physics Laboratories \\ Harvard University \\ Cambridge, MA 02138, USA \\ June 1994 \\ (revised: September 1994)
}

\begin{abstract}
The low energy structure of a theory containing light and heavy particle species which are separated by a mass gap can adequately be described by an effective theory which contains only the light particles. In this work we present a thorough analysis of the effective field theory of the linear $O(N)$ sigma model in the spontaneously broken phase. In particular, we present a detailed discussion of two techniques, a shortdistance expansion and a method based on loop-integrals, which can be used to explicitly evaluate the functional relationships between the low energy constants of the effective theory and the parameters of the underlying theory. We furthermore provide a detailed analysis of the matching relation between the linear sigma model and its effective theory, in order to clarify some discrepancies which can be found in the literature.
\end{abstract}

\footnotetext{
${ }^{1}$ Work supported in part by Deutsche Forschungsgemeinschaft, by Schweizerischer Nationalfonds, and by NSF grant Phy-92-18167.
} 


\section{Introduction}

The effective field theory of the linear sigma model has recently received considerable attention in the analysis of the symmetry breaking sector of the electroweak interaction. So far the agreement between experimental data and the standard model predictions is almost perfect. Yet, the precise nature of the spontaneous breakdown of the electroweak symmetry remains unknown and various scenarios are discussed in the literature, including such different theories as a heavy Higgs [1, 2, 3, 4] or technicolor models [5]. One way out of this ignorance is to replace the Higgs sector of the standard model by a model independent parametrization which provides a unified description of the low energy physics in the symmetry breaking sector, i.e. up to energies of the order of $1 \mathrm{TeV}$. If all the unknown particles of the underlying theory, e.g. a Higgs or a Technirho, are heavy, physics at low energies is dominated by the Goldstone bosons arising from spontaneous symmetry breaking, and all the other known particles of the standard model. In this case a convenient description is provided by replacing the linear sigma model in the standard model Lagrangian by an effective Lagrangian which contains only the Goldstone bosons [1, 6]. The unknown physics of the symmetry breaking sector is then hidden in the low-energy constants which occur in the effective Lagrangian. Thus, if the relations between the constants in the effective Lagrangian and the parameters of the underlying theory are known, low energy physics can provide interesting information about the precise nature of the spontaneous symmetry breaking which may occur at a comparatively high energy scale, such as in technicolor models.

In this work, we present a thorough analysis of the effective field theory description of the linear $O(N)$ sigma model in the spontaneously broken phase. Our goal is twofold. On the one hand, we provide a detailed discussion of the matching relation between the linear sigma model and its effective theory. A formal definition of the effective theory as an adequate representation of the full theory in the low energy region will certainly require that corresponding Green's functions in both theories have the same low energy structure. It is known [7, 8] that literally integrating out only the heavy degrees of freedom in the full theory generally does not yield an effective Lagrangian which

meets this requirement. Our discussion of this point is intended to clarify the discrepancies which can be found in the literature [9, 2] on the effective theory of the linear sigma model. On the other hand, we want to go beyond 
a formal definition and provide a detailed discussion of two techniques which can be used to explicitly evaluate the effective Lagrangian. The first one employs a short distance expansion in configuration-space, while the second one is based on the evaluation of loop-integrals in momentum-space. For the case we are considering here the result for the effective Lagrangian can already be found in the literature [9]. The derivation given there, however, is rather sketchy and does not discuss the steps involved in any detail. The present article contains a thorough account of the problem. Furthermore it focuses on the discussion of the general techniques which can be used to evaluate the effective Lagrangian for a given underlying theory, provided the theory shows a mass gap between heavy and light particles and the coupling is weak in the low energy region.

The outline of this work is as follows: In the next section we present a brief review of the linear sigma model. In section 3 the general low energy structure of the effective Lagrangian is discussed, exploiting only the symmetry properties of the full theory. In the following section we provide a thorough analysis of the matching relation between both theories which includes an exact definition of the effective Lagrangian. In the next three sections the detailed description of two methods to calculate the low-energy constants at order $p^{4}$ can be found. In section 8 we discuss renormalization and express the bare quantities in terms of physical parameters. Finally we summarize this work in section 9 .

\section{The Linear $O(N)$ Sigma Model}

In this section we employ the technique used in ref. [9] from which the abundant literature on the linear sigma model may be traced.

In the absence of external fields the Lagrangian of the $O(N)$ symmetric linear sigma model is given by

$$
\frac{1}{2} \partial_{\mu} \phi^{T} \partial^{\mu} \phi+\frac{1}{2} m^{2} \phi^{T} \phi-\frac{g}{4}\left(\phi^{T} \phi\right)^{2}
$$

where $\phi^{A}$ is an $N$-component field. For $m^{2}>0$ the classical potential has

its minimum at a nonzero value $\phi^{T} \phi=m^{2} / g$ and the $O(N)$ symmetry is spontaneously broken to $O(N-1)$. Accordingly, the $N$-component field $\phi^{A}$ describes 1 massive (pseudo) scalar and $N-1$ massless Goldstone bosons. 
In order to obtain the generating functional for the Green's functions we couple the field $\phi^{A}$ to a set of external fields with spin 0 and spin 1:

$$
\mathcal{L}_{\sigma}=\frac{1}{2} \nabla_{\mu} \phi^{T} \nabla^{\mu} \phi+\frac{1}{2} m^{2} \phi^{T} \phi-\frac{g}{4}\left(\phi^{T} \phi\right)^{2}+f^{T} \phi+h \operatorname{tr} F_{\mu \nu} F^{\mu \nu} .
$$

The spin 1 field is defined in terms of the generators $T^{\alpha}$ of the Lie algebra $o(N)$ and the $N(N-1) / 2$ gauge fields $F_{\mu}^{\alpha}$,

$$
F_{\mu}^{A B}=\left(T^{\alpha}\right)^{A B} F_{\mu}^{\alpha},
$$

which are coupled covariantly to the scalar field,

$$
\nabla_{\mu} \phi^{A}=\partial_{\mu} \phi^{A}+F_{\mu}^{A B} \phi^{B}
$$

The generating functional $W_{\sigma}\left[F_{\mu}, f\right]$ is defined by a path integral

$$
e^{i W_{\sigma}\left[F_{\mu}, f\right]}=\int \mathrm{d} \mu[\phi] e^{i \int \mathrm{d}^{d} x \mathcal{L}_{\sigma}} .
$$

Derivatives of this functional with respect to the spin 0 field $f^{A}$ generate Green's functions of the scalar fields $\phi^{A}$ while derivatives with respect to the spin 1 field $F_{\mu}^{\alpha}$ generate Green's functions of the currents,

$$
J_{\mu}^{\alpha}=\left(\partial_{\mu} \phi\right)^{T} T^{\alpha} \phi
$$

The last term in the Lagrangian is the trace over the square of the field strength $F_{\mu \nu}^{A B}$ associated with the nonabelian gauge field $F_{\mu}^{A B}$,

$$
F_{\mu \nu}=\left[\nabla_{\mu}, \nabla_{\nu}\right]
$$

In the absence of the spin 1 field $F_{\mu}$ the generating functional $W_{\sigma}[0, f]$ can be rendered finite by a proper renormalization of the mass $m$, the coupling constant $g$, and the scalar fields $\phi^{A}$ and $f^{A}$. Green's functions of the currents, on the other hand, are more singular at short distances than Green's functions of the fields. The time ordering of current operators gives rise to ambiguities which do not occur for the fields themselves. The corresponding Green's functions are unique only up to contact terms. This ambiguity is reflected by the presence of the last term in the Lagrangian (2.2), which enters Green's functions of the currents through contact terms. The corresponding constant 
$h$ can be renormalized such that the full generating functional $W_{\sigma}\left[F_{\mu}, f\right]$ remains finite after the regulator is removed.

The generating functional defined in eq. (2.5) has two interesting properties. First, note that both the generating functional $W_{\sigma}$ and the Lagrangian $\mathcal{L}_{\sigma}$ are gauge invariant under local $O(N)$ transformations of the form

$$
\begin{aligned}
\phi & \rightarrow V \phi \\
f & \rightarrow V f \\
F_{\mu} & \rightarrow V F_{\mu} V^{T}+V \partial_{\mu} V^{T} .
\end{aligned}
$$

This statement is equivalent to the Ward identities of the linear sigma model. Second, this description also allows for the presence of an explicitly symmetry breaking term of the form $c^{T} \phi$ which provides the Goldstone bosons with a mass proportional to $\sqrt{|c|}$. Keeping this constant small enough, one still has a mass gap between the Goldstone bosons and the heavy particle. To obtain Green's functions in this case one merely has to expand the generating functional around the nonzero value $f=c$ instead of $f=0$. Except for some minor changes our analysis remains valid for arbitrary values of the constant $c$, as long as a mass gap is maintained between the Goldstone bosons and the heavy particle.

Let us pause for a moment and consider the particular case $N=4$ which describes the symmetry breaking sector in the standard model of the electroweak interaction. The group $O(4)$ is semi-simple, $O(4) \approx S U(2) \times S U(2)$, and one can express the nonabelian gauge field $F_{\mu}$ in terms of external vector and axial-vector fields:

$$
\begin{aligned}
F_{\mu}^{0 i} & =a_{\mu}^{i} \\
F_{\mu}^{i j} & =-\epsilon^{i j k} v_{\mu}^{k} .
\end{aligned}
$$

Using the method of steepest decent, the one-loop approximation to the generating functional is given by

$$
W_{\sigma}\left[F_{\mu}, f\right]=\int \mathrm{d}^{d} x \mathcal{L}_{\sigma}\left(\phi_{0}, \partial_{\mu} \phi_{0}, F_{\nu}, f\right)+\frac{i}{2} \operatorname{lndet} \tilde{D}
$$

where $\tilde{D}$ is the differential operator

$$
(y, \tilde{D} y)=\int \mathrm{d}^{d} x y^{T}\left(\nabla_{\mu} \nabla^{\mu}+\tilde{\sigma}\right) y
$$


with

$$
\tilde{\sigma}^{A B}=\left(-m^{2}+g \phi_{0}^{T} \phi_{0}\right) \delta^{A B}+2 g \phi_{0}^{A} \phi_{0}^{B} .
$$

The field $\phi_{0}^{A}$ is a solution of the classical equations of motion

$$
\nabla_{\mu} \nabla^{\mu} \phi_{0}=f+m^{2} \phi_{0}-g\left(\phi_{0}^{T} \phi_{0}\right) \phi_{0}
$$

The one-loop approximation given in eq. (2.11) represents the first two terms in the expansion of the generating functional in powers of $\hbar$. This corresponds to expansions of the Green's functions in powers of the coupling constant $g$. In the next step we will analyze the low energy structure of this approximation in the range of small energies and momenta, i.e., below the (physical) mass $M$ of the heavy particle. This will in addition introduce an expansion in powers of momenta $p^{2} / M^{2}$. Since we want to describe the low energy structure of the linear sigma model in terms of an effective Lagrangian which contains only the Goldstone bosons, the following parametrization of the classical solution $\phi_{0}^{A}$ will turn out to be useful:

$$
\phi_{0}^{A} \doteq \frac{m}{\sqrt{g}} R U^{A}, \quad U^{T} U=1
$$

The massless modes are now represented by the $N$-component vector $U^{A}$, confined to the sphere $S_{N-1}$ while the massive mode is described by the radial variable $R$. It is well known [10], that the coordinates of the coset space related to the spontaneous symmetry breaking pattern, i.e. the full group factored by the unbroken subgroup, provide a convenient representation for the Goldstone boson fields in the effective Lagrangian. In our case the coset space is $O(N) / O(N-1)$ which is isomorphic to the sphere $S_{N-1}$. Thus, the definition (2.15) turns out to be quite obvious.

In terms of the new field the minimum of the classical potential is located at $R=1$ and the equations of motion (2.14) can be rewritten in the form

$$
\begin{aligned}
\square R+R\left(U^{T} \nabla_{\mu} \nabla^{\mu} U\right) & =\chi_{0}^{T} U+m^{2} R\left(1-R^{2}\right) \\
R\left(\nabla_{\mu} \nabla^{\mu} U-U\left(U^{T} \nabla_{\mu} \nabla^{\mu} U\right)\right) & =\chi_{0}-U\left(\chi_{0}^{T} U\right)-2 \partial_{\mu} R \nabla^{\mu} U,
\end{aligned}
$$

where

$$
\chi_{0} \doteq \frac{\sqrt{g}}{m} f .
$$


The radial variable $R$ describes a massive fluctuation around $R=1$ and its value at a particular space-time point depends only on the behaviour of the external field in a small neighbourhood of this point. Thus, for slowly varying external fields the behaviour of the massive mode is under control and the equation of motion (2.16) can be solved algebraically. The result is an expansion in powers of derivatives of the external fields:

$$
R=1+\delta R_{1}+\delta R_{2}+\ldots, \quad \delta R_{n}=\mathcal{O}\left(p^{2 n}\right) .
$$

Note that the field $f$ counts as a quantity of order $p^{2}$ while the gauge field $F_{\mu}$ occurring in the covariant derivative is of order $p$. $U$ is a quantity of order 1 .

The first two nontrivial terms of this series are readily evaluated to be

$$
\begin{aligned}
& \delta R_{1}=\frac{1}{2 m^{2}}\left[\chi_{0}^{T} U+\nabla_{\mu} U^{T} \nabla^{\mu} U\right] \\
& \delta R_{2}=-\frac{3}{2} \delta R_{1}^{2}+\frac{1}{2 m^{2}} \delta R_{1}\left(\nabla_{\mu} U^{T} \nabla^{\mu} U\right)+\text { total derivative } .
\end{aligned}
$$

The tree-level contribution to the generating functional is given by

$$
\frac{1}{2} \frac{m^{2}}{g} \int \mathrm{d}^{d} x\left(R\left(\chi_{0}^{T} U\right)+\frac{1}{2} m^{2} R^{4}\right)+h \int \mathrm{d}^{d} x \operatorname{tr} F_{\mu \nu} F^{\mu \nu}
$$

Thus, with the help of expansion (2.19) one is able to determine the low energy behaviour of the tree-level contribution to the generating functional to any required order in $p^{2}$.

Finally, by changing to a new basis, we separate the massless fluctuations around the classical solution $\phi_{0}^{A}$, which are bound to the sphere $U^{T} U=1$, from the massive fluctuation along $U^{A}$ :

$$
\phi^{A}-\phi_{0}^{A}=\xi U^{A}+\sum_{i=1}^{N-1} \epsilon_{i}^{A} \eta^{i},
$$

with

$$
\epsilon_{i}^{T} \epsilon_{j}=\delta_{i j}, \quad \epsilon_{i}^{T} U=0
$$

and with the completeness relation

$$
U^{A} U^{B}+\sum_{i=1}^{N-1} \epsilon_{i}^{A} \epsilon_{i}^{B}=\delta^{A B} .
$$


In order to describe the action of the differential operator $\tilde{D}$ in the new basis of the mass eigenstates we introduce the following notation:

$$
\begin{aligned}
d & =\square+2 m^{2}-f_{\mu}^{T} f^{\mu}+3 m^{2}\left(R^{2}-1\right) \\
f_{i}^{\mu} & =U^{T} \nabla^{\mu} \epsilon_{i} \\
D_{\mu} & =\partial_{\mu}+\Gamma_{\mu} \\
\Gamma_{\mu}^{i j} & =\epsilon_{i}^{T} \nabla_{\mu} \epsilon_{j} \\
\delta & =\left(D_{\mu} f^{\mu}\right)+2 f_{\mu}^{T} D^{\mu} \\
\left(\eta, \delta^{T} \xi\right) & =(\delta \eta, \xi) \\
D & =D_{\mu} D^{\mu}+\sigma \\
\sigma^{i j} & =-f_{\mu}^{i} f^{j \mu}+m^{2}\left(R^{2}-1\right) \delta^{i j} .
\end{aligned}
$$

One finds

$$
(y, \tilde{D} y)=(\xi, d \xi)+(\xi, \delta \eta)+\left(\delta^{T} \xi, \eta\right)+(\eta, D \eta)
$$

To separate massless and massive fluctuations we furthermore diagonalize this expression with the transformation $\hat{\xi} \doteq \xi+d^{-1} \delta \eta$ :

$$
(y, \tilde{D} y)=(\hat{\xi}, d \hat{\xi})+\left(\eta,\left(D-\delta^{T} d^{-1} \delta\right) \eta\right)
$$

Thus, we obtain the following representation for the one-loop contribution to the generating functional

$$
\ln \operatorname{det} \tilde{D}=\operatorname{lndet} D+\operatorname{lndet} d+\ln \operatorname{det}\left(1-D^{-1} \delta^{T} d^{-1} \delta\right) \text {. }
$$

\section{The Effective Lagrangian}

In this section we will analyze the general low energy structure of the linear $O(N)$ sigma model. We will set up the effective Lagrangian description for the generating functional exploiting only the symmetry properties of the linear sigma model. Thus, this discussion is completely general and applies to any underlying theory which has the same symmetry breaking pattern and a mass gap between the Goldstone bosons and the heavier particle species. To begin the discussion, let us recall that the generating functional $W_{\sigma}\left[F_{\mu}, f\right]$ as defined in the previous section is gauge invariant under local $O(N)$ transformations as given in eq. (2.8). Due to the presence of Goldstone bosons 
generated by spontaneous symmetry breaking, the structure of the Green's functions is nontrivial even at low energies. Only the contributions from the massive modes admit a Taylor series expansion. It is known 11] that, if the underlying theory (the linear sigma model in our case) does not contain anomalies and is Lorentz invariant, the low energy structure of the generating functional can be described in terms of an effective field theory with a symmetric effective Lagrangian.

In our case, this effective Lagrangian depends on an $N$-component field $U^{A}$ confined to the sphere $U^{T} U=1$ which describes the $N-1$ Goldstone bosons and which is coupled to the external fields $f^{A}$ and $F_{\mu}^{A B}$. The latter is coupled to the $O(N)$ vector $U^{A}$ by the covariant derivative given in eq. (2.4). The effective Lagrangian is the most general functional invariant under Lorentz transformations and the gauge transformations given in eq. (2.8),

$$
\mathcal{L}_{e f f}=\mathcal{L}_{e f f}\left(U, \nabla_{\mu} U, \nabla_{\mu} \nabla_{\nu} U, f, \ldots\right) .
$$

The Lagrangian $\mathcal{L}_{\text {eff }}$ is a sum of terms with an increasing number of derivatives, corresponding to an expansion in powers of the momentum,

$$
\mathcal{L}_{\text {eff }}=\mathcal{L}_{2}+\mathcal{L}_{4}+\mathcal{L}_{6}+\ldots
$$

where $\mathcal{L}_{i}$ is of order $p^{i}$. (If explicitly symmetry breaking terms are present, this expansion includes powers of the Goldstone boson masses as well.) The generating functional is defined as the path integral

$$
e^{i W_{e f f}\left[F_{\mu}, f\right]}=\int \mathrm{d} \mu[U] e^{i \int \mathrm{d}^{d} x \mathcal{L}_{e f f}} .
$$

In general, there are two different kinds of contributions to the generating functional. On the one hand, we have tree-level contributions, given by the integral $\int \mathrm{d}^{d} x \mathcal{L}_{\text {eff }}$, which has to be evaluated at the stationary point, i.e. with the solutions of the equations of motion. One the other hand there are contributions from loops, which ensure unitarity. General power counting arguments show [12], that $n$-loop corrections are suppressed by powers $p^{2 n}$ as compared to the tree-level. Thus, one-loop corrections with vertices of $\mathcal{L}_{2}$ are of order $p^{4}$ while those with vertices of $\mathcal{L}_{4}$ are of order $p^{6}$, as are two-loop corrections. The suppression of loops by powers of $p^{2}$ which allows the perturbative study of the systematic expansion in powers of momenta is 
related to the fact that Goldstone bosons do not interact at threshold. Thus, at order $p^{4}$ the generating functional $W_{\text {eff }}\left[F_{\mu}, f\right]$ is given by

$$
W_{\text {eff }}\left[F_{\mu}, f\right]=\int \mathrm{d}^{d} x\left(\mathcal{L}_{2}+\mathcal{L}_{4}\right)+\frac{i}{2} \ln \operatorname{det} \bar{D}+\mathcal{O}\left(p^{6}\right)
$$

where the term lndet $\bar{D}$ describes the one-loop corrections with vertices from $\mathcal{L}_{2}$.

The leading term of the generating functional is of order $p^{2}$ and given by the tree-level contributions of $\mathcal{L}_{2}$

$$
W_{e f f}\left[F_{\mu}, f\right]=\int \mathrm{d}^{d} x \mathcal{L}_{2}+\mathcal{O}\left(p^{4}\right)
$$

Imposing Lorentz invariance and taking account of the identities $U^{T} \nabla_{\mu} U=0$ and $U^{T} \nabla_{\mu} \nabla^{\mu} U=-\nabla_{\mu} U^{T} \nabla^{\mu} U$, the most general effective Lagrangian at order $p^{2}$ which is invariant under local $O(N)$ transformations involves only two low energy constants and is given by

$$
c_{1} \nabla_{\mu} U^{T} \nabla^{\mu} U+c_{2} f^{T} U
$$

In view of eq. (2.18) it will turn out to be convenient to introduce another field $\chi^{A}$ proportional to $f^{A}$ and define this Lagrangian in the form

$$
\mathcal{L}_{2}=F^{2}\left(\frac{1}{2} \nabla_{\mu} U^{T} \nabla^{\mu} U+\chi^{T} U\right)
$$

Accordingly, we will replace the dependence of the generating functional $W_{\text {eff }}$ and of the effective Lagrangian $\mathcal{L}_{\text {eff }}$ on the field $f^{A}$ by the dependence on the field $\chi^{A}$. The action in eq. (3.5) is to be evaluated with the solution $\bar{U}^{A}$ of the classical equations of motion

$$
\nabla_{\mu} \nabla^{\mu} \bar{U}-\bar{U}\left(\bar{U}^{T} \nabla_{\mu} \nabla^{\mu} \bar{U}\right)=\chi-\bar{U}\left(\chi^{T} \bar{U}\right)
$$

Note that this solution coincides with the solution $U^{A}$ of eq. (2.17) only at leading order. The corrections $U^{A}-\bar{U}^{A}$ are due to the massive mode and admit an expansion in powers of $p^{2}$ (see below).

To obtain the one-loop contribution of order $p^{4}$ we again use the method of steepest descent and parametrize the fluctuations around the solution $\bar{U}^{A}$ 
of the equations of motion in a way similar to the one used in the previous section on the linear sigma model,

$$
U^{A}-\bar{U}^{A}=\xi^{i} \bar{\epsilon}_{i}^{A}-\frac{1}{2} \xi^{i} \xi^{i} \bar{U}^{A}+\ldots
$$

The vectors $\left\{\bar{U}^{A}, \bar{\epsilon}_{i}^{A}\right\}$ are orthonormal, and thus obey relations as given in eqs. (2.24,2.25). The presence of the second term in eq. (3.9) is necessary to ensure that both vectors, $\bar{U}^{A}$ and $U^{A}$, are of unit length. Introducing a notation similar to the definitions (2.26 2.33),

$$
\begin{aligned}
\bar{f}_{i}^{\mu} & =\bar{U}^{T} \nabla^{\mu} \bar{\epsilon}_{i} \\
\bar{D}_{\mu} & =\partial_{\mu}+\bar{\Gamma}_{\mu} \\
\bar{\Gamma}_{\mu}^{i j} & =\bar{\epsilon}_{i}^{T} \nabla_{\mu} \bar{\epsilon}_{j} \\
\bar{\sigma}^{i j} & =-f_{\mu}^{i} f^{j \mu}+\left(\nabla_{\mu} \bar{U}^{T} \nabla^{\mu} \bar{U}+\chi^{T} \bar{U}\right) \delta^{i j},
\end{aligned}
$$

the differential operator $\bar{D}$ in eq. (3.4) turns out to be

$$
\bar{D}=\bar{D}_{\mu} \bar{D}^{\mu}+\bar{\sigma}
$$

Finally, we have to specify the general effective Lagrangian at order $p^{4}$. Note that the most general effective Lagrangian at this order is given as a linear combination of a maximal set of independent $O(N)$ invariant terms of order $p^{4}$. On the one hand, redundant terms can be eliminated by using relations of the form

$$
\int \mathrm{d}^{d} x \nabla_{\nu} \nabla_{\mu} U^{T} \nabla^{\mu} \nabla^{\nu} U=\int \mathrm{d}^{d} x \nabla_{\mu} \nabla^{\mu} U^{T} \nabla_{\nu} \nabla^{\nu} U+\nabla_{\nu} U^{T} F^{\nu \mu} \nabla_{\mu} U,
$$

which are readily verified using the definition of the field strength given in eq. (2.7). On the other hand, the Lagrangian $\mathcal{L}_{4}$ contributes only at the classical level. Hence, the equations of motion (3.8) can also be used to eliminate further redundant terms. From eq. (3.8) one can infer the following two identities

$$
\begin{aligned}
\left(\chi^{T} \nabla_{\mu} \nabla^{\mu} \bar{U}\right)+\left(\chi^{T} \bar{U}\right)\left(\nabla_{\mu} \bar{U}^{T} \nabla^{\mu} \bar{U}\right)+\left(\chi^{T} \bar{U}\right)^{2} & =\chi^{T} \chi \\
\left(\nabla_{\mu} \nabla^{\mu} \bar{U}^{T} \nabla_{\nu} \nabla^{\nu} \bar{U}\right)-\left(\nabla_{\mu} \bar{U}^{T} \nabla^{\mu} \bar{U}\right)^{2}+\left(\chi^{T} \bar{U}\right)^{2} & =\chi^{T} \chi
\end{aligned}
$$


The general effective Lagrangian at order $p^{4}$ contains eight independent low energy constants and can be brought into the form

$$
\begin{aligned}
\mathcal{L}_{4}=d_{1} & \left(\nabla_{\mu} \bar{U}^{T} \nabla^{\mu} \bar{U}\right)^{2}+d_{2}\left(\nabla_{\mu} \bar{U}^{T} \nabla_{\nu} \bar{U}\right)\left(\nabla^{\mu} \bar{U}^{T} \nabla^{\nu} \bar{U}\right) \\
& +d_{3}\left(\chi^{T} \bar{U}\right)^{2}+d_{4}\left(\chi^{T} \bar{U}\right)\left(\nabla_{\mu} \bar{U}^{T} \nabla^{\mu} \bar{U}\right) \\
& +d_{5}\left(\bar{U}^{T} F_{\mu \nu} F^{\mu \nu} \bar{U}\right)+d_{6}\left(\nabla_{\nu} \bar{U}^{T} F^{\nu \mu} \nabla_{\mu} \bar{U}\right) \\
& +d_{7}\left(\chi^{T} \chi\right)+d_{8} \operatorname{tr}\left(F_{\mu \nu} F^{\mu \nu}\right) .
\end{aligned}
$$

\section{Matching}

In section two we have determined the generating functional of the linear sigma model up to one loop. In the next section we have described the low energy structure of this generating functional in terms of an effective Lagrangian which contains only the $N-1$ Goldstone bosons. Exploiting the symmetry properties of the underlying theory one can reduce the number of low energy constants that are involved in the effective Lagrangian to two at order $p^{2}$ and to eight at order $p^{4}$. All these low energy constants are determined by the underlying theory. To express the low energy constants in terms of the parameters of the linear sigma model we require that both theories yield the same Green's functions in the low energy region:

$$
W_{\sigma}\left[F_{\mu}, f\right]=W_{e f f}\left[F_{\mu}, \chi\right] .
$$

Recall that we have absorbed one of the low energy constants in section 3 by introducing the external scalar field $\chi^{A}$. Thus eq. (4.1) determines the relation between the scalar fields $\chi^{A}$ and $f^{A}$ as well. Using eqs. (2.11, 2.36) and (3.4) we obtain at order $p^{4}$ :

$$
\begin{gathered}
\int \mathrm{d}^{d} x \mathcal{L}_{\sigma}+\frac{i}{2} \operatorname{lndet} D+\frac{i}{2} \ln \operatorname{det} d+\frac{i}{2} \operatorname{lndet}\left(1-D^{-1} \delta^{T} d^{-1} \delta\right) \\
=\int \mathrm{d}^{d} x\left(\mathcal{L}_{2}+\mathcal{L}_{4}\right)+\frac{i}{2} \ln \operatorname{let} \bar{D} .
\end{gathered}
$$

We will solve this equation for the low energy constants in a couple of steps. To begin with, let us recall that both theories, the linear sigma model and the effective theory, contain Goldstone bosons. Thus, both sides of eq. (4.2) should reproduce all singularities associated with the light particles 
and the corresponding contributions should cancel. This is indeed the case. At order $p^{4}$ all of these singularities are described by the determinants of the two differential operators $D$ and $\bar{D}$. A glance at eqs. (2.17, 3.8) shows that there is only a small difference between these operators, related to the fact that the fields $U^{A}$ and $\bar{U}^{A}$ satisfy slightly different equations of motion. We will, however, see below, that the corresponding difference between $\ln \operatorname{det} D$ and lndet $\bar{D}$ is of higher order and can be neglected in our analysis. Thus, at order $p^{4}$ our matching relation simplifies to

$$
\int \mathrm{d}^{d} x \mathcal{L}_{\sigma}+\frac{i}{2} \ln \operatorname{det} d+\frac{i}{2} \ln \operatorname{det}\left(1-D^{-1} \delta^{T} d^{-1} \delta\right)=\int \mathrm{d}^{d} x\left(\mathcal{L}_{2}+\mathcal{L}_{4}\right) .
$$

Note that the two determinants on the left hand side of this equation are still nonlocal objects, while the right hand side is a local expression. The operator $d$, however, is related to the heavy particle in the linear sigma model and its determinant admits an expansion in terms of local quantities involving an increasing number of derivatives, which correspond to an expansion in powers of momenta. The second determinant on the left hand side of eq. (4.3) is a more complicated matter. Since it involves the operator $D$ of the massless modes it is not a purely local object. The analysis in the following reveals that its nonlocality in the low-energy region shows up at the order $p^{6}$. Thus, at the order $p^{4}$ all terms in the low energy expansion of the determinants in eq. (4.3) are local. The comparison of corresponding coefficients on both sides of this equation yields the functional relationship between the low energy constants and the parameters of the linear sigma model at this order.

If one were to determine the low energy constants up to the order $p^{6}$, the generating functionals of the linear sigma model and of the effective theory would have to be calculated up to the two-loop level. In this case, additional terms would occur which ensure that the matching condition contains only local terms up to the order $p^{6}$. This will be explained in greater detail below.

Before we use eq. (4.3) to determine the low-energy constants up to the order $p^{4}$, some further comments on our matching relation (4.1) are in order. The crucial point to notice here is that the expansion of the nonlocal terms in eq. (4.3) takes place after all integrations over the fields $U$ and $R$ have been performed. More explicitly, this amounts to a relation between the full path integrals, i.e.

$$
\int \mathrm{d} \mu[U] e^{i \int \mathrm{d}^{d} x \mathcal{L}_{e f f}\left(U, F_{\mu}, \chi\right)}=\int \mathrm{d} \mu[\phi] e^{i \int \mathrm{d}^{d} x \mathcal{L}_{\sigma}\left(\phi, F_{\mu}, f\right)} .
$$


where $\mathcal{L}_{\text {eff }}=\mathcal{L}_{2}+\mathcal{L}_{4}$ at order $p^{4}$. To get a better understanding of this point, let us define the quantity $\Gamma_{e f f}\left[U, F_{\mu}, f\right]$ (as in ref. [2]) by performing the path integral on the right hand side of eq. (4.4) only over the massive particle,

$$
e^{i \Gamma_{e f f}\left[U, F_{\mu}, f\right]} \doteq \int \mathrm{d} \mu[R] e^{i \int \mathrm{d}^{d} x \mathcal{L}_{\sigma}\left(R, U, F_{\mu}, f\right)},
$$

where we have used the parametrization (2.15) for the field $\phi^{A}$ as well as the identity

$$
\mathrm{d} \mu[\phi]=\mathrm{d} \mu[U] \mathrm{d} \mu[R] .
$$

Our matching relation can thus be rewritten in the form

$$
\int \mathrm{d} \mu[U] e^{i \int \mathrm{d}^{d} x \mathcal{L}_{e f f}\left(U, F_{\mu}, \chi\right)}=\int \mathrm{d} \mu[U] e^{i \Gamma_{e f f}\left[U, F_{\mu}, f\right]} .
$$

Note that the quantity $\Gamma_{e f f}\left[U, F_{\mu}, f\right]$ itself is a nonlocal object. Since it involves only the propagation of the heavy particle, it certainly admits an expansion in terms of local quantities as well. Such an expansion would introduce another density $L_{e f f}$, defined by

$$
\int \mathrm{d}^{d} x L_{e f f}=\Gamma_{e f f} .
$$

However, in eq. (4.7), which defines the effective Lagrangian $\mathcal{L}_{\text {eff }}$, one first integrates over the massless modes $U$ and then expands the resulting nonlocal contributions, as explained in the sequel of eq. (4.3). One may now pose the question of whether it is permissible to change the order of these two operations. In that case one would first expand the integrand on the right hand side of eq. (4.7), and then perform the integration over the massless modes. If that procedure were correct, one would obtain the result that $L_{\text {eff }}$ and $\mathcal{L}_{\text {eff }}$ are the same and our matching relation (4.7) between integrals could be replaced be an equivalent relation between integrands, i.e.

$$
e^{i \int \mathrm{d}^{d} x \mathcal{L}_{e f f}\left(U, F_{\mu}, \chi\right)}=\int \mathrm{d} \mu[R] e^{i \int \mathrm{d}^{d} x \mathcal{L}_{\sigma}\left(R, U, F_{\mu}, f\right)} .
$$

At the classical level this relation is obviously correct, since then

$$
\int \mathrm{d}^{d} x \mathcal{L}_{e f f}=\int \mathrm{d}^{d} x L_{e f f}=\Gamma_{e f f}=\int \mathrm{d}^{d} x \mathcal{L}_{\sigma},
$$


where $\mathcal{L}_{\sigma}$ is to be evaluated with the classical solution $R$ of the equations of motion (2.16). However, if quantum corrections are taken into account, it is generally wrong. Using the method of steepest decent, we again expand the field $R$ as

$$
R=R_{0}+z,
$$

where $R_{0}$ satisfies the equations of motion (2.16). The measure is given by

$$
\mathrm{d} \mu[R]=\mathcal{N} \prod_{x} R^{N-1} \mathrm{~d} R=\mathcal{N} \prod_{x} R_{0}^{N-1}(1+\mathcal{O}(z)) \mathrm{d} z .
$$

Furthermore, in the dimensional regularization scheme the factor $R_{0}^{N-1}$ in the measure does not contribute to the integral. Thus, at the order we are considering here, the measure is given by

$$
\mathrm{d} \mu[R]=\mathcal{N} \prod_{x} \mathrm{~d} z,
$$

and one obtains

$$
\int \mathrm{d}^{d} x \mathcal{L}_{\sigma}+\frac{i}{2} \ln \operatorname{let} d=\int \mathrm{d}^{d} x L_{e f f} .
$$

Now one can compare the results for $L_{\text {eff }}$ and $\mathcal{L}_{2}+\mathcal{L}_{4}$ as given in eqs. (4.14) and (4.3). At order $p^{4}$ they both receive contributions from loop integrals which include only propagators of massive particles, represented by the term Indet $d$. In addition to that, however, $\mathcal{L}_{2}+\mathcal{L}_{4}$ furthermore receives contributions from loop integrals which include propagators of both, massive and massless particles, as described by the last term on the left hand side of eq. (4.3). These contributions are missing in the representation (4.14) of $L_{\text {eff }}$. Thus, if quantum corrections are taken into account, one must require equality between the full integrals, as in our matching condition (4.1). In general this requirement cannot be replaced by a relation between integrands. In other words, the integration over the massless modes and the expansion of the nonlocal objects do not commute. The relevance of mixed loops containing propagators of light and heavy particles is also discussed in the framework of QED in ref. [8]. 


\section{Short-Distance Expansion}

In this section we will discuss the evaluation of the low-energy constants from eq. (4.3) with the help of short-distance expansions. The first term on the left hand side of eq. (4.3), which describes all tree-level contributions, is readily evaluated with the help of eqs. (2.19,2.20,2.21)

$$
\begin{aligned}
& \int \mathrm{d}^{d} x \mathcal{L}_{\sigma}=h \int \mathrm{d}^{d} x \operatorname{tr}\left(F_{\mu \nu} F^{\mu \nu}\right) \\
& +\left(\frac{m^{2}}{g}\right) \int \mathrm{d}^{d} x\left[\frac{1}{2} \nabla_{\mu} U^{T} \nabla^{\mu} U+\chi_{0}^{T} U\right] \\
& +\left(\frac{1}{4 g}\right) \int \mathrm{d}^{d} x\left[\left(\nabla_{\mu} U^{T} \nabla^{\mu} U\right)^{2}+2\left(\nabla_{\mu} U^{T} \nabla^{\mu} U\right)\left(\chi_{0}^{T} U\right)+\left(\chi_{0}^{T} U\right)^{2}\right] .
\end{aligned}
$$

The second term on the left hand side of eq. (4.3) describes all those one-loop corrections which involve only heavy particles. We expand this contribution in powers of the difference $\sigma_{M}=d-\left(\square+2 m^{2}\right)$ which counts as a quantity of order $p^{2}$. At order $p^{4}$ we get

$$
\frac{i}{2} \operatorname{lndet} d=\frac{i}{2} \operatorname{lndet} d_{0}+\frac{i}{2} \operatorname{Tr}\left[d_{0}^{-1} \sigma_{M}\right]-\frac{i}{4} \operatorname{Tr}\left[d_{0}^{-1} \sigma_{M}\right]^{2},
$$

with $d_{0}=\square+2 m^{2}$. The second term is given by

$$
\frac{i}{2} G_{M}(0) \int \mathrm{d}^{d} x \sigma_{M}(x),
$$

where $G_{M}(z)$ is the Feynman propagator of a scalar particle with mass $M=$ $\sqrt{2} m$. The third term is of the form

$-\frac{i}{4} \int \mathrm{d}^{d} x \mathrm{~d}^{d} y \sigma_{M}(x) \sigma_{M}(x+y) G_{M}^{2}(y)=-\frac{i}{4} \int \mathrm{d}^{d} x \sigma_{M}^{2}(x) \int \mathrm{d}^{d} y G_{M}^{2}(y)+\mathcal{O}\left(p^{6}\right)$.

Note that only the local behaviour of the slowly varying external fields is relevant since the massive propagator cuts off large distances. Using eqs. (2.20,2.21,2.26) we obtain the following relevant contribution

$$
\begin{aligned}
\frac{i}{2} \operatorname{lndet} d= & -2 m^{2} \hat{\lambda}_{0}\left(2 m^{2}\right) \int \mathrm{d}^{d} x\left[2\left(\nabla_{\mu} U^{T} \nabla^{\mu} U\right)+3\left(\chi_{0}^{T} U\right)\right] \\
& -\left(2 \hat{\lambda}_{0}\left(2 m^{2}\right)+\frac{1}{16 \pi^{2}}\right) \int \mathrm{d}^{d} x\left(\nabla_{\mu} U^{T} \nabla^{\mu} U\right)^{2}
\end{aligned}
$$




$$
\begin{aligned}
& -\left(3 \hat{\lambda}_{0}\left(2 m^{2}\right)+\frac{3}{16 \pi^{2}}\right) \int \mathrm{d}^{d} x\left(\nabla_{\mu} U^{T} \nabla^{\mu} U\right)\left(\chi_{0}^{T} U\right) \\
& -\left(\frac{3}{2} \hat{\lambda}_{0}\left(2 m^{2}\right)+\frac{9}{64 \pi^{2}}\right) \int \mathrm{d}^{d} x\left(\chi_{0}^{T} U\right)^{2}
\end{aligned}
$$

where

$$
\begin{aligned}
\hat{\lambda}_{0}\left(M^{2}\right) & \doteq \lambda_{0}+\frac{1}{32 \pi^{2}} \ln \frac{M^{2}}{\mu^{2}} \\
\lambda_{0} & \doteq \frac{1}{16 \pi^{2}} \mu^{d-4}\left(\frac{1}{d-4}-\frac{1}{2}\left(\ln 4 \pi+\Gamma^{\prime}(1)+1\right)\right) .
\end{aligned}
$$

This leaves us with the last term on the left hand side of eq. (4.3) which is the hardest to evaluate. If we again expand the localized quantity $\left\langle x\left|d^{-1}\right| y\right\rangle$ in powers of $\sigma_{M}=d-\left(\square+2 m^{2}\right)$, only the first three terms contribute at order $p^{4}$

$$
\begin{aligned}
& \frac{i}{2} \operatorname{lndet}\left(1-D^{-1} \delta^{T} d^{-1} \delta\right)=-\frac{i}{2} \operatorname{Tr}\left[\delta D^{-1} \delta^{T} d_{0}^{-1}\right] \\
& \quad+\frac{i}{2} \operatorname{Tr}\left[\delta D^{-1} \delta^{T} d_{0}^{-1} \sigma_{M} d_{0}^{-1}\right]-\frac{i}{4} \operatorname{Tr}\left[\delta D^{-1} \delta^{T} d_{0}^{-1}\right]^{2},
\end{aligned}
$$

where $d_{0}=\square+2 m^{2}$. Furthermore, for the last two terms only the leading singularity of the kernel $\left\langle x\left|\delta D^{-1} \delta^{T}\right| y\right\rangle$, determined by the d'Alembertian, is relevant at this order. In particular, the relevant contribution to the second term is given by

$$
\begin{aligned}
& -2 i \int \mathrm{d}^{d} x \mathrm{~d}^{d} y \mathrm{~d}^{d} z f_{\mu}^{T}(x) f_{\nu}(x+y) \sigma_{M}(x+z) \partial^{\mu} \partial^{\nu} G_{0}(y) G_{M}(y-z) G_{M}(z) \\
& =-2 i \int \mathrm{d}^{d} x f_{\mu}^{T}(x) f_{\nu}(x) \sigma_{M}(x) \int \mathrm{d}^{d} y \mathrm{~d}^{d} z \partial^{\mu} \partial^{\nu} G_{0}(y) G_{M}(y-z) G_{M}(z)+\mathcal{O}\left(p^{6}\right),
\end{aligned}
$$

where $G_{0}(z)$ is the Feynman propagator of a massless particle. Again the massive propagator cuts off large distances. Lorentz invariance requires this contribution to be of the form

$$
-\frac{2 i}{d} \int \mathrm{d}^{d} x f_{\mu}^{T} f^{\mu} \sigma_{M} \int \mathrm{d}^{d} y G_{M}^{2}(y) .
$$

In the same way, we obtain the following result for the relevant contribution to the third term in eq. (5.8)

$$
-4 i \int \mathrm{d}^{d} x\left(f_{\mu}^{T} f_{\nu}\right)\left(f_{\rho}^{T} f_{\sigma}\right) \int \mathrm{d}^{d} y \mathrm{~d}^{d} z \mathrm{~d}^{d} v \partial^{\mu} \partial^{\nu} G_{0}(y) G_{M}(y-z) \partial^{\rho} \partial^{\sigma} G_{0}(z-v) G_{M}(v)
$$




$$
=-\frac{4 i}{d(d+2)} \int \mathrm{d}^{d} x\left(\left(f_{\mu}^{T} f^{\mu}\right)\left(f_{\nu}^{T} f^{\nu}\right)+2\left(f_{\mu}^{T} f^{\nu}\right)\left(f_{\nu}^{T} f^{\mu}\right)\right) \int \mathrm{d}^{d} y G_{M}^{2}(y)
$$

Using the identity

$$
f_{\mu}^{T} f_{\nu}=\nabla_{\mu} U^{T} \nabla_{\nu} U
$$

we get at order $p^{4}$

$$
\begin{aligned}
& \frac{i}{2} \operatorname{Tr}\left[\delta D^{-1} \delta^{T} d_{0}^{-1} \sigma_{M} d_{0}^{-1}\right]-\frac{i}{4} \operatorname{Tr}\left[\delta D^{-1} \delta^{T} d_{0}^{-1}\right]^{2}= \\
& -\left(\frac{7}{3} \hat{\lambda}_{0}\left(2 m^{2}\right)+\frac{1}{(4 \pi)^{2}} \frac{19}{36}\right) \int \mathrm{d}^{d} x\left(\nabla^{\mu} U^{T} \nabla_{\mu} U\right)^{2} \\
& -\left(\frac{2}{3} \hat{\lambda}_{0}\left(2 m^{2}\right)+\frac{1}{(4 \pi)^{2}} \frac{1}{18}\right) \int \mathrm{d}^{d} x\left(\nabla^{\mu} U^{T} \nabla^{\nu} U\right)\left(\nabla_{\mu} U^{T} \nabla_{\nu} U\right) \\
& \quad-\left(3 \hat{\lambda}_{0}\left(2 m^{2}\right)+\frac{1}{(4 \pi)^{2}} \frac{3}{4}\right) \int \mathrm{d}^{d} x\left(\nabla^{\mu} U^{T} \nabla_{\mu} U\right)\left(\chi_{0}^{T} U\right) .
\end{aligned}
$$

The first term on the right hand side of eq. (5.8) is more complicated, because the next-to-leading singularity of the kernel $\left\langle x\left|\delta D^{-1} \delta^{T}\right| y\right\rangle$ is relevant in this case as well. The computation of the corresponding contribution, which may be based on either the short distance expansion or the calculation of two-, three- and four-point functions, can be found in the next two sections. Both methods yield the following result at order $p^{4}$

$$
\begin{aligned}
\frac{-i}{2} \operatorname{Tr} & {\left[\delta D^{-1} \delta^{T} d_{0}^{-1}\right]=-2 m^{2}\left(\hat{\lambda}_{0}\left(2 m^{2}\right)-\frac{1}{(4 \pi)^{2}} \frac{1}{4}\right) \int \mathrm{d}^{d} x \nabla_{\mu} U^{T} \nabla^{\mu} U } \\
- & \left(\frac{4}{3} \hat{\lambda}_{0}\left(2 m^{2}\right)-\frac{1}{(4 \pi)^{2}} \frac{5}{9}\right) \int \mathrm{d}^{d} x\left(\nabla^{\mu} U^{T} \nabla_{\mu} U\right)^{2} \\
& +\left(\frac{4}{3} \hat{\lambda}_{0}\left(2 m^{2}\right)-\frac{1}{(4 \pi)^{2}} \frac{5}{9}\right) \int \mathrm{d}^{d} x\left(\nabla^{\mu} U^{T} \nabla^{\nu} U\right)\left(\nabla_{\mu} U^{T} \nabla_{\nu} U\right) \\
& -\left(\hat{\lambda}_{0}\left(2 m^{2}\right)-\frac{1}{(4 \pi)^{2}} \frac{1}{4}\right) \int \mathrm{d}^{d} x\left(\chi_{0}^{T} U\right)\left(\nabla^{\mu} U^{T} \nabla_{\mu} U\right) \\
& -\frac{1}{(4 \pi)^{2}} \frac{1}{12} \int \mathrm{d}^{d} x\left(\chi_{0}^{T} U\right)^{2} \\
& -\left(\frac{1}{6} \hat{\lambda}_{0}\left(2 m^{2}\right)+\frac{1}{(4 \pi)^{2}} \frac{1}{72}\right) \int \mathrm{d}^{d} x U^{T} F_{\mu \nu} F^{\mu \nu} U
\end{aligned}
$$




$$
\begin{aligned}
& -\left(\frac{1}{3} \hat{\lambda}_{0}\left(2 m^{2}\right)-\frac{1}{(4 \pi)^{2}} \frac{11}{36}\right) \int \mathrm{d}^{d} x \nabla_{\mu} U^{T} F^{\mu \nu} \nabla_{\nu} U \\
& +\frac{1}{(4 \pi)^{2}} \frac{1}{12} \int \mathrm{d}^{d} x \chi_{0}^{T} \chi_{0} .
\end{aligned}
$$

The expressions in eqs. (5.1,5.5, 5.13,5.14) provide the complete result for the expansion of the one-loop approximation to the generating functional of the linear sigma model in powers of the momentum up to the order $p^{4}$. Now one can read off the relations between the low energy constants and the parameters of the linear sigma model. The contributions of order $p^{2}$ occurring in eqs. (5.1,5.5, 5.14), which have to be matched with the effective Lagrangian $\mathcal{L}_{2}$ given in eq. (3.7), can be brought into the form

$$
F^{2} \int \mathrm{d}^{d} x\left[\frac{1}{2}\left(\nabla_{\mu} U^{T} \nabla^{\mu} U\right)+\chi^{T} U\right]
$$

provided that we set

$$
\begin{aligned}
F^{2} & =\left(\frac{m^{2}}{g}\right)\left[1-g\left(12 \hat{\lambda}_{0}\left(2 m^{2}\right)-\frac{1}{(4 \pi)^{2}}\right)\right] \\
\chi & =\chi_{0}\left[1+g\left(6 \hat{\lambda}_{0}\left(2 m^{2}\right)-\frac{1}{(4 \pi)^{2}}\right)\right] .
\end{aligned}
$$

The relation between $\chi$ and $\chi_{0}$ then allows one to determine the remaining low energy constants by matching the contributions of order $p^{4}$ occurring in eqs. (3.18) with the effective Lagrangian $\mathcal{L}_{4}$ :

$$
\begin{aligned}
& d_{1}=\frac{1}{4 g}-\frac{17}{3} \hat{\lambda}_{0}\left(2 m^{2}\right)-\frac{1}{(4 \pi)^{2}} \frac{35}{36} \\
& d_{2}=\frac{2}{3} \hat{\lambda}_{0}\left(2 m^{2}\right)-\frac{1}{(4 \pi)^{2}} \frac{11}{18} \\
& d_{3}=\frac{1}{4 g}-\frac{9}{2} \hat{\lambda}_{0}\left(2 m^{2}\right)-\frac{1}{(4 \pi)^{2}} \frac{11}{6} \\
& d_{4}=\frac{1}{2 g}-10 \hat{\lambda}_{0}\left(2 m^{2}\right)-\frac{3}{(4 \pi)^{2}} \\
& d_{5}=-\frac{1}{6} \hat{\lambda}_{0}\left(2 m^{2}\right)-\frac{1}{(4 \pi)^{2}} \frac{1}{72}
\end{aligned}
$$




$$
\begin{aligned}
d_{6} & =-\frac{1}{3} \hat{\lambda}_{0}\left(2 m^{2}\right)+\frac{1}{(4 \pi)^{2}} \frac{11}{36} \\
d_{7} & =\frac{1}{(4 \pi)^{2}} \frac{1}{12} \\
d_{8} & =h .
\end{aligned}
$$

Finally, we have to show that it was indeed consistent to neglect all corrections which are due to the difference $U^{A}-\bar{U}^{A}$. By comparing the equations of motion for $U^{A}$ and $\bar{U}^{A}$ given in eqs. (2.17,3.8) one infers that $U^{A}-\bar{U}^{A}$ receives contributions of order $p^{2}$, due to the fact that $R \neq 1$, and also of order $g$, since $\chi \neq \chi_{0}$. First of all we can use the relation (5.17) to express $\chi_{0}$ by $\chi$ everywhere in the generating functional $W_{\sigma}$. The replacement of $U^{A}$ by $\bar{U}^{A}$ can lead to corrections of order $p^{4}$ only in the following contribution to the generating functional:

$$
\begin{aligned}
\Sigma[U, F, \chi] & \doteq \int \mathrm{d}^{d} x \frac{F^{2}}{2}\left(\nabla_{\mu} U^{T} \nabla^{\mu} U+2 \chi^{T} U\right) \\
& +\left(\frac{1}{4 g}\right) \int \mathrm{d}^{d} x\left(\nabla_{\mu} U^{T} \nabla^{\mu} U+\chi^{T} U\right)^{2} .
\end{aligned}
$$

In all the remaining terms one can safely replace $U^{A}$ by $\bar{U}^{A}$, since the corresponding corrections to the generating functional are of the order $g$ and $p^{6}$ which is beyond the accuracy of our calculation. In order to show that

$$
\Sigma[U, F, \chi]=\Sigma[\bar{U}, F, \chi]+\mathcal{O}(g)+\mathcal{O}\left(p^{6}\right)
$$

we make an intermediate step to separate the expansions in $p^{2}$ and in $g$. Let $V^{A}$ be the stationary point of the functional $\Sigma[V, F, \chi]$, subject to the condition $V^{T} V=1$. Since $U^{A}$ itself extremizes the functional $\Sigma\left[U, m / \sqrt{g}, \chi_{0}\right]$, it can easily be seen that $U^{A}-V^{A}=\mathcal{O}(g)$ and, therefore,

$$
\Sigma[U, F, \chi]=\Sigma[V, F, \chi]+\mathcal{O}(g)
$$

since at the stationary point only corrections proportional to the square of $U-V$ can enter. Similarly, because $\bar{U}^{A}$ extremizes the action of the nonlinear sigma model (cf. eq. (3.7)) we have $\bar{U}^{A}-V^{A}=\mathcal{O}\left(p^{2}\right)$, from which we get

$$
\Sigma[\bar{U}, F, \chi]=\Sigma[V, F, \chi]+\mathcal{O}\left(p^{6}\right) .
$$


Putting eqs. (5.21) and (5.22) together we have proven the claim in eq. (5.20). The main point in the whole analysis is that, at the stationary point, the replacement of $V$ by $V+\delta V$ can only lead to corrections which are of second order in $\delta V$.

\section{Employing the Heat Kernel}

In Euclidean space the short distance properties of the differential operator $D$,

$$
D=-D_{\mu} D_{\mu}+\sigma, \quad D_{\mu}=\partial_{\mu}+\Gamma_{\mu},
$$

are governed by the Laplacian $\square$. In $d$-dimensional Euclidean space one has

$$
\left\langle x\left|e^{\lambda \square}\right| y\right\rangle=(4 \pi \lambda)^{-d / 2} e^{-z^{2} / 4 \lambda},
$$

where $z=x-y$. In order to determine the properties of the operator $D$ at short distances, we define 13

$$
\left\langle x\left|e^{-\lambda D}\right| y\right\rangle=(4 \pi \lambda)^{-d / 2} e^{-z^{2} / 4 \lambda} H(x|\lambda| y) .
$$

The heat kernel $H(x|\lambda| y)$ satisfies the differential equation

$$
\left(\frac{\partial}{\partial \lambda}+\frac{1}{\lambda} z_{\mu} D_{\mu}-D_{\mu} D_{\mu}+\sigma\right) H(x|\lambda| y)=0
$$

with the boundary condition

$$
H(x|0| x)=1 .
$$

Inserting the Taylor expansion

$$
H(x|\lambda| y)=\sum_{n=0}^{\infty} \lambda^{n} H_{n}(x \mid y)
$$

into the differential equation (6.4), one obtains the following recursive relations for the heat coefficients $H_{n}$ :

$$
\begin{array}{r}
\left(1+n+z_{\mu} \overrightarrow{D_{\mu}}\right) H_{n+1}+\left(-\overrightarrow{D_{\mu}} \vec{D}_{\mu}+\sigma\right) H_{n}=0 \\
z_{\mu} \overrightarrow{D_{\mu}} H_{0}=0 .
\end{array}
$$


Note that the covariant derivative $D_{\mu}$ which appears on the left hand side of the heat coefficients acts to the right on the space coordinate $x$. In the foregoing equation we have shown this explicitly by the arrows. In the following we will furthermore use the covariant derivative $\overleftarrow{D}_{\mu}$

$$
\overleftarrow{D}_{\mu} \doteq \overleftarrow{\partial}_{\mu}-\Gamma_{\mu}
$$

which will always appear on the right hand side of the heat coefficients and act to the left on the coordinate $y$. Applying the derivatives $\vec{D}_{\mu}$ and $\overleftarrow{D_{\mu}}$ repeatedly to the relations in eq. (6.7) one obtains the following results for the heat coefficients

$$
\begin{aligned}
H_{0}(x \mid x) & =1 \\
H_{1}(x \mid x) & =-\sigma \\
H_{2}(x \mid x) & =\frac{1}{12} \Gamma_{\mu \nu} \Gamma_{\mu \nu}+\frac{1}{2} \sigma^{2}-\frac{1}{6}\left[D_{\mu},\left[D_{\mu}, \sigma\right]\right] \\
\left.\left(\vec{D}_{\mu} H_{0}\right)\right|_{x=y} & =\left.\left(H_{0} \overleftarrow{D}_{\nu}\right)\right|_{x=y}=0 \\
\left.\left(\vec{D}_{\mu} \vec{D}_{\nu} H_{0}\right)\right|_{x=y} & =\left.\left(\vec{D}_{\mu} H_{0} \overleftarrow{D}_{\nu}\right)\right|_{x=y}=\frac{1}{2} \Gamma_{\mu \nu},
\end{aligned}
$$

where

$$
\Gamma_{\mu \nu} \doteq\left[D_{\mu}, D_{\nu}\right] .
$$

The kernel $\left\langle x\left|\delta D^{-1} \delta^{T}\right| y\right\rangle$ occurring in the traces of eq. (5.8) can be rewritten in the form

$$
\left\langle x\left|\delta D^{-1} \delta^{T}\right| y\right\rangle=\left(\left(D_{\mu} f_{\mu}\right)^{T}+2 f_{\mu}^{T} \vec{D}_{\mu}\right)_{x}\left\langle x\left|D^{-1}\right| y\right\rangle\left(2 \stackrel{\overleftarrow{D}}{\mathbb{D}_{\nu}} f_{\nu}+\left(D_{\nu} f_{\nu}\right)\right)_{y}
$$

which in turn can be expressed in terms of the heat kernel, due to the relation

$$
\left\langle x\left|D^{-1}\right| y\right\rangle=\int_{0}^{\infty} \frac{\mathrm{d} \lambda}{(4 \pi \lambda)^{d / 2}} e^{-z^{2} / 4 \lambda} H(x|\lambda| y) .
$$

It is convenient to define yet another kernel $h(x|\lambda| y)$ by

$$
\left\langle x\left|\delta D^{-1} \delta^{T}\right| y\right\rangle \doteq \int_{0}^{\infty} \frac{\mathrm{d} \lambda}{(4 \pi \lambda)^{d / 2}} e^{-z^{2} / 4 \lambda} h(x|\lambda| y) .
$$


If we use the following representation for the massive propagator,

$$
G_{M}(z)=\int_{0}^{\infty} \frac{\mathrm{d} \rho}{(4 \pi \rho)^{d / 2}} e^{\frac{-z^{2}}{4 \rho}-2 m^{2} \rho},
$$

the first term on the right hand side of eq. (5.8) can be brought to the form

$$
\operatorname{Tr}\left[\delta D^{-1} \delta^{T} d_{0}^{-1}\right]=\frac{1}{(4 \pi)^{d}} \int_{0}^{\infty} \frac{\mathrm{d} \lambda \mathrm{d} \rho}{\lambda^{d / 2} \rho^{d / 2}} \int \mathrm{d}^{d} x \mathrm{~d}^{d} y e^{-\frac{z^{2}}{4}\left(\frac{1}{\lambda}+\frac{1}{\rho}\right)-2 m^{2} \rho} h(x|\lambda| y) .
$$

The kernel $h(x|\lambda| y)$ is readily determined from eqs. 6.3,6.15, 6.16, 6.17) and turns out to be

$$
\begin{aligned}
& h(x|\lambda| y)=2 f_{\mu}^{T}\left(\vec{D}_{\mu} H\right)\left(D_{\nu} f_{\nu}\right)+2\left(D_{\mu} f_{\mu}\right)^{T}\left(H \stackrel{\leftarrow}{D_{\nu}}\right) f_{\nu} \\
& +4 f_{\mu}^{T}\left(\vec{D}_{\mu} H \stackrel{\leftarrow}{D_{\nu}}\right) f_{\nu}+\left(D_{\mu} f_{\mu}\right)^{T} H\left(D_{\nu} f_{\nu}\right)+\frac{2}{\lambda} f_{\mu}^{T} H f_{\mu} \\
& \quad-\frac{z_{\mu}}{\lambda}\left\{2 f_{\mu}^{T}\left(H \stackrel{\leftarrow}{D_{\nu}}\right) f_{\nu}-2 f_{\nu}^{T}\left(\vec{D}_{\nu} H\right) f_{\mu}+f_{\mu}^{T} H\left(D_{\nu} f_{\nu}\right)\right. \\
& \left.-\left(D_{\nu} f_{\nu}\right)^{T} H f_{\mu}\right\}-\frac{z_{\mu} z_{\nu}}{\lambda^{2}} f_{\mu}^{T} H f_{\nu} .
\end{aligned}
$$

Note that in this equation all factors appearing on the right hand side of the heat kernel depend on the space coordinate $y$ while all factors appearing on the left hand side depend on $x$. The exponential factor in the integrand in eq. (6.19) cuts off contributions from large values of $z$. Since the kernel $h(x|\lambda| y)$ is a smooth function for slowly varying external fields, it admits a Taylor expansion in powers of $z$. The expansion of the last term in eq. (6.20), for example, can be written in the form

$$
\begin{aligned}
f_{\mu}^{T}(x) & H(x|\lambda| y) f_{\nu}(y) \\
= & \left.\left(f_{\mu}^{T} H f_{\nu}\right)\right|_{x=y}+\left.z_{\rho}\left(\left(D_{\rho} f_{\mu}\right)^{T} H f_{\nu}+f_{\mu}^{T}\left(\vec{D}_{\rho} H\right) f_{\nu}\right)\right|_{x=y} \\
& \quad+\left.\frac{z_{\rho} z_{\sigma}}{2}\left(\left(D_{\rho} D_{\sigma} f_{\mu}\right)^{T} H f_{\nu}+2\left(D_{\rho} f_{\mu}\right)^{T}\left(\vec{D}_{\sigma} H\right) f_{\nu}+f_{\mu}^{T}\left(\vec{D}_{\rho} \vec{D}_{\sigma} H\right) f_{\nu}\right)\right|_{x=y} \\
& \quad+\ldots
\end{aligned}
$$

If we furthermore insert the Taylor expansion (6.6) for the heat kernel and keep only terms which are relevant at order $p^{4}$ we obtain

$$
h(x|\lambda| y)=\left(D_{\mu} f_{\mu}\right)^{T} H_{0}\left(D_{\nu} f_{\nu}\right)+4 f_{\mu}^{T}\left(\vec{D}_{\mu} H_{0} \stackrel{\leftarrow}{D_{\nu}}\right) f_{\nu}
$$




$$
\begin{aligned}
& +\frac{2}{\lambda} f_{\mu}^{T} H_{0} f_{\mu}+2 f_{\mu}^{T} H_{1} f_{\mu}+\frac{z_{\rho} z_{\sigma}}{\lambda}\left[\left(D_{\rho} D_{\sigma} f_{\mu}\right)^{T} H_{0} f_{\mu}+f_{\mu}^{T}\left(\overrightarrow{D_{\rho}} \vec{D}_{\sigma} H_{0}\right) f_{\mu}\right] \\
& -\frac{z_{\mu} z_{\rho}}{\lambda}\left[\left(D_{\rho} f_{\mu}\right)^{T} H_{0}\left(D_{\nu} f_{\nu}\right)-\left(D_{\rho} D_{\nu} f_{\nu}\right)^{T} H_{0} f_{\mu}\right. \\
& \left.\quad+2 f_{\mu}^{T}\left(\vec{D}_{\rho} H_{0} \overleftarrow{D_{\nu}}\right) f_{\nu}-2 f_{\nu}^{T}\left(\overrightarrow{D_{\rho}} \vec{D}_{\nu} H_{0}\right) f_{\mu}\right] \\
& -\frac{z_{\mu} z_{\nu}}{\lambda^{2}} f_{\mu}^{T} H_{0} f_{\nu}-\frac{z_{\mu} z_{\nu}}{\lambda} f_{\mu}^{T} H_{1} f_{\nu} \\
& -\frac{z_{\mu} z_{\nu} z_{\rho} z_{\sigma}}{2 \lambda^{2}}\left[\left(D_{\rho} D_{\sigma} f_{\mu}\right)^{T} H_{0} f_{\nu}+f_{\mu}^{T}\left(\vec{D}_{\rho} \vec{D}_{\sigma} H_{0}\right) f_{\nu}\right] .
\end{aligned}
$$

Note that we have already omitted all terms that are odd in $z$ or contain one of the factors given in eq. (6.12) because they will drop out anyway. Now one can perform the Gaussian integration over $z$ and use the relations (6.9) to (6.13) for the heat coefficients to obtain (up to partial integrations)

$$
\begin{aligned}
\operatorname{Tr}[ & \left.\delta D^{-1} \delta^{T} d_{0}^{-1}\right]=\frac{1}{(4 \pi)^{d / 2}} \int \mathrm{d}^{d} x \int_{0}^{\infty} \frac{\mathrm{d} \lambda \mathrm{d} \rho}{(\lambda+\rho)^{d / 2}} e^{-2 m^{2} \rho} \\
& {\left[2 f_{\mu}^{T} \Gamma_{\mu \nu} f_{\nu}-2 f_{\mu}^{T} \sigma f_{\mu}+\left(D_{\mu} f_{\mu}\right)^{T}\left(D_{\nu} f_{\nu}\right)+\frac{2}{\lambda+\rho} f_{\mu}^{T} f_{\mu}\right.} \\
& -\frac{2 \rho}{(\lambda+\rho)}\left\{\left(D_{\mu} f_{\nu}\right)^{T}\left(D_{\mu} f_{\nu}\right)+2\left(D_{\mu} f_{\mu}\right)^{T}\left(D_{\nu} f_{\nu}\right)+2 f_{\mu}^{T} \Gamma_{\mu \nu} f_{\nu}-f_{\mu}^{T} \sigma f_{\mu}\right\} \\
& \left.+\frac{2 \rho^{2}}{(\lambda+\rho)^{2}}\left\{\left(D_{\mu} f_{\nu}\right)^{T}\left(D_{\mu} f_{\nu}\right)+\left(D_{\nu} f_{\mu}\right)^{T}\left(D_{\mu} f_{\nu}\right)+\left(D_{\mu} f_{\mu}\right)^{T}\left(D_{\nu} f_{\nu}\right)\right\}\right]
\end{aligned}
$$

To bring this result into the form of eq. (5.14) we make use of the relation given in eq. (5.12) as well as of the following identity

$$
\left(D_{\mu} f_{\nu}\right)^{T}\left(D_{\rho} f_{\sigma}\right)=\nabla_{\mu} \nabla_{\nu} U^{T} \nabla_{\rho} \nabla_{\sigma} U-\left(U^{T} \nabla_{\mu} \nabla_{\nu} U\right)\left(U^{T} \nabla_{\rho} \nabla_{\sigma} U\right) .
$$

Note that some of the terms in this equation do not occur in the effective Lagrangian given in eq. (3.18). These terms can be expressed in terms of the complete set of independent invariants that occur in the Lagrangian. In order to eliminate redundant terms we proceed as discussed at the end of section 3. Using the definition of the field strength $F_{\mu \nu}$ given in eq. (2.7) one can eliminate the various dependent terms of the form $\nabla_{\mu} \nabla_{\nu} U^{T} \nabla_{\rho} \nabla_{\sigma} U$ in favour of only $\nabla_{\mu} \nabla_{\mu} U^{T} \nabla_{\nu} \nabla_{\nu} U$ and some other terms, that are already present in the effective Lagrangian. Since we have furthermore used the 
equations of motion of the effective Lagrangian to reduce the number of independent terms in $\mathcal{L}_{4}$, we should do the same in this case as well. From the equation of motion (2.17) of the linear sigma model we infer a relation similar to the one given in eq. (3.17),

$$
\nabla_{\mu} \nabla_{\mu} U^{T} \nabla_{\nu} \nabla_{\nu} U-\left(\nabla_{\mu} U^{T} \nabla_{\mu} U\right)^{2}-\chi_{0}^{T} \chi_{0}+\left(\chi_{0}^{T} U\right)^{2}=0,
$$

which holds up to corrections of order $p^{6}$ which can be neglected. Thus, using the identities $6.24,6.25$ ) to eliminate all terms of the form $\nabla_{\mu} \nabla_{\nu} U^{T} \nabla_{\rho} \nabla_{\sigma} U$ we obtain the result given in eq. (5.14).

\section{Loops}

In the preceding two sections we have evaluated all one-loop contributions in the matching relation (4.3) which determine the low energy constants of the effective Lagrangian at order $p^{4}$ by means of the short-distance expansion in configuration space. In this section we want to compare this method with the evaluation by means of loop-integrals in momentum space. We shall see that at order $p^{4}$ this involves tadpole graphs and two-, three-, and four-point functions. Since we want to present a general comparison between these two methods - short-distance expansion versus loop-integrals - we will not discuss all contributions in detail.

First, we will discuss the evaluation of the second term on the left hand side of eq. (4.3) which can be expanded as shown in eq. (5.2). The second term on the right hand side of this equation is a simple tadpole graph of the massive propagator as shown explicitly in eq. (5.3). The third term in eq. (5.2) involves a two-point function with two massive propagators. In momentum-space it is given by (cf. eq. (5.4))

$$
-\frac{i}{4} \int \frac{d^{d} p_{1}}{(2 \pi)^{d}} \sigma_{M}\left(p_{1}\right) \sigma_{M}\left(-p_{1}\right) \int \frac{d^{d} p}{(2 \pi)^{d}} \frac{1}{\left(M^{2}-p^{2}\right)\left(M^{2}-\left(p+p_{1}\right)^{2}\right)} .
$$

The loop-integral is readily evaluated and is regular at $p_{1}=0$. Hence, it admits an expansion for small momenta,

$$
\int \frac{d^{d} p}{(2 \pi)^{d}} \frac{1}{\left(M^{2}-p^{2}\right)\left(M^{2}-\left(p+p_{1}\right)^{2}\right)}
$$




$$
\begin{aligned}
& =-i\left(2 \hat{\lambda}_{0}\left(M^{2}\right)-\frac{1}{16 \pi^{2}}\left\{\rho \ln \left(\frac{\rho-1}{\rho+1}\right)+1\right\}\right) \\
& =-i\left(2 \hat{\lambda}_{0}\left(M^{2}\right)+\frac{1}{16 \pi^{2}}\right)+\mathcal{O}\left(p_{1}^{2}\right),
\end{aligned}
$$

where

$$
\rho \doteq \sqrt{1-\frac{4 M^{2}}{p_{1}^{2}}}
$$

At order $p^{4}$ only the leading term of the expansion of this two-point function is relevant. Thus one obtains the same result as given in eq. (5.4). This may be compared with the short-distance expansion used in eq. (5.4), where the integrand $\sigma_{M}(x) \sigma_{M}(x+y)$ is expanded in $y$. In both cases only the leading term is relevant at order $p^{4}$.

To discuss the evaluation of the contributions from the third term on the left hand side of eq. (4.3), it is convenient to introduce the following shorthand notation for the $n$-point functions. We define the general two-point function by

$$
B_{\mu_{1} \ldots \mu_{n}}\left(p_{1} ; m_{1}, m_{2}\right) \doteq \frac{1}{i} \int \frac{d^{d} p}{(2 \pi)^{d}} \frac{p_{\mu_{1}} \ldots p_{\mu_{n}}}{\left(m_{1}^{2}-p^{2}\right)\left(m_{2}^{2}-\left(p+p_{1}\right)^{2}\right)} .
$$

The function $B\left(p_{1} ; m_{1}, m_{2}\right)$ denotes the integral where no momenta appear in the numerator, e.g. $B\left(p_{1} ; M, M\right)$ is given explicitly in eq. (7.2). Similarly we denote the general three-point function by $C_{\mu_{1} \ldots \mu_{n}}\left(p_{1}, p_{2} ; m_{1}, m_{2}, m_{3}\right)$ and the general four-point function by $D_{\mu_{1} \ldots \mu_{n}}\left(p_{1}, p_{2}, p_{3} ; m_{1}, m_{2}, m_{3}, m_{4}\right)$.

Whereas the kernel $\left\langle x\left|d^{-1}\right| y\right\rangle$ is related to the propagator $G_{M}$ of the massive particle, the kernel $\left\langle x\left|D^{-1}\right| y\right\rangle$, which enters in eq. (5.8), leads to propagators $G_{0}$ of the massless particles in the loops. We can formally rewrite the operator $D$ in the form (cf. eq. (2.32))

$$
D \doteq \square\left(1+\square^{-1}\left(\left(D_{\mu} \Gamma^{\mu}\right)+2 \Gamma_{\mu} \partial^{\mu}+\sigma\right)\right) \text {. }
$$

Therefore we get

$$
\begin{aligned}
& \left\langle x\left|\left(D^{-1}\right)^{i k}\right| y\right\rangle=\delta^{i k} G_{0}(x-y) \\
& \quad-\quad \int \mathrm{d}^{d} z G_{0}(x-z)\left(\left(D_{\mu} \Gamma^{\mu}\right)^{i k}(z)+2 \Gamma_{\mu}^{i k}(z) \partial^{\mu}+\sigma^{i k}(z)\right) G_{0}(z-y) \\
& \quad+4 \int \mathrm{d}^{d} z \mathrm{~d}^{d} u \Gamma_{\mu}^{i j}(z) \Gamma_{\nu}^{j k}(u) G_{0}(x-z) \partial^{\mu} G_{0}(z-u) \partial^{\nu} G_{0}(u-y)+\ldots .
\end{aligned}
$$


In the last two traces on the right hand side of eq. (5.8) only the first term in expansion (7.7) is relevant. Note that this term also determines the leading singularity of the kernel $\left\langle x\left|\delta D^{-1} \delta^{T}\right| y\right\rangle$. The relevant contribution to the second term on the right hand side of eq. (5.8) is given by the integral (cf. eq. (5.9))

$$
-2 \int \frac{d^{d} p_{1}}{(2 \pi)^{d}} \frac{d^{d} p_{2}}{(2 \pi)^{d}} f^{\mu T}\left(p_{1}+p_{2}\right) f^{\nu}\left(-p_{1}\right) \sigma_{M}\left(-p_{2}\right) C_{\mu \nu}\left(p_{1}, p_{2} ; 0, M, M\right),
$$

which involves two massive and one massless propagator. The loop-integral which contributes to the third term in eq. (5.8) involves two massive and two massless propagators and is of the form (cf. eq. (5.11))

$$
\begin{gathered}
4 \int \frac{d^{d} p_{1}}{(2 \pi)^{d}} \frac{d^{d} p_{2}}{(2 \pi)^{d}} \frac{d^{d} p_{3}}{(2 \pi)^{d}} f^{\mu T}\left(p_{1}+p_{2}+p_{3}\right) f^{\nu}\left(-p_{1}\right) f^{\rho T}\left(-p_{2}\right) f^{\sigma}\left(-p_{3}\right) \\
D_{\mu \nu \rho \sigma}\left(p_{1}, p_{2}, p_{3} ; 0, M, 0, M\right) .
\end{gathered}
$$

In expressions (7.8) and (7.9) the external fields account already for four powers in the momenta so that only the leading terms in the expansions of the function $C_{\mu \nu}\left(p_{1}, p_{2} ; 0, M, M\right)$ and $D_{\mu \nu \rho \sigma}\left(p_{1}, p_{2}, p_{3} ; 0, M, 0, M\right)$, which are of order 1 , are relevant. Using the expressions for the leading terms as given in the appendix, one obtains the same results as given in eq. (5.13).

The evaluation of the first term on the right hand side of eq. (5.8) by means of the short distance expansion was more complicated, because in this case the next-to-leading singularity of the kernel $\left\langle x\left|\delta D^{-1} \delta^{T}\right| y\right\rangle$ is relevant as well. In the language of loop-integrals this translates to the fact, that all three terms in expansion (7.7) of the kernel $\left\langle x\left|D^{-1}\right| y\right\rangle$ need to be taken into account. Hence, at order $p^{4}$ the first term on the right hand side of eq. (5.8) receives contributions from two-, three-, and four-point functions. In particular, one obtains

$$
\begin{gathered}
\frac{1}{2} \int \frac{d^{d} p_{1}}{(2 \pi)^{d}}\left\{b^{T}\left(p_{1}\right) a\left(-p_{1}\right) B\left(p_{1} ; 0, M\right)+2 i b^{T}\left(p_{1}\right) f_{\mu}\left(-p_{1}\right) B^{\mu}\left(p_{1} ; 0, M\right)\right. \\
\left.-2 i f_{\mu}^{T}\left(p_{1}\right) a\left(-p_{1}\right) B^{\mu}\left(p_{1} ; 0, M\right)+4 f_{\mu}^{T}\left(p_{1}\right) f_{\nu}\left(-p_{1}\right) B^{\mu \nu}\left(p_{1} ; 0, M\right)\right\} \\
-2 \int \frac{d^{d} p_{1}}{(2 \pi)^{d}} \frac{d^{d} p_{2}}{(2 \pi)^{d}}\left\{\left[f_{\mu}^{T}\left(p_{1}+p_{2}\right)\left(\left(D_{\rho} \Gamma^{\rho}\right)\left(-p_{1}\right)+\sigma\left(-p_{1}\right)\right) f_{\nu}\left(-p_{2}\right)\right.\right.
\end{gathered}
$$




$$
\begin{aligned}
& +b^{T}\left(p_{1}+p_{2}\right) \Gamma_{\mu}\left(-p_{1}\right) f_{\nu}\left(-p_{2}\right) \\
& \left.-f_{\mu}^{T}\left(p_{1}+p_{2}\right) \Gamma_{\nu}\left(-p_{1}\right) a\left(-p_{2}\right)\right] C^{\mu \nu}\left(p_{1}, p_{2} ; 0,0, M\right) \\
& -2 i f_{\mu}^{T}\left(p_{1}+p_{2}\right) \Gamma_{\nu}\left(-p_{1}\right) f_{\rho}\left(-p_{2}\right)\left[C^{\mu \nu \rho}\left(p_{1}, p_{2} ; 0,0, M\right)\right. \\
& \left.\left.+p_{1}^{\nu} C^{\mu \rho}\left(p_{1}, p_{2} ; 0,0, M\right)+p_{1}^{\rho} C^{\mu \nu}\left(p_{1}, p_{2} ; 0,0, M\right)\right]\right\}
\end{aligned}
$$

and

$$
\begin{gathered}
-8 \int \frac{d^{d} p_{1}}{(2 \pi)^{d}} \frac{d^{d} p_{2}}{(2 \pi)^{d}} \frac{d^{d} p_{3}}{(2 \pi)^{d}} f_{\mu}^{T}\left(p_{1}+p_{2}+p_{3}\right) \Gamma_{\nu}\left(-p_{1}\right) \Gamma_{\rho}\left(-p_{2}\right) f_{\sigma}\left(-p_{3}\right) \\
D^{\mu \nu \rho \sigma}\left(p_{1}, p_{2}, p_{3} ; 0,0,0, M\right),
\end{gathered}
$$

where

$$
a=\left(D_{\mu} f^{\mu}\right)-2 \Gamma_{\mu} f^{\mu}, \quad b^{T}=\left(D_{\mu} f^{\mu}\right)^{T}+2 f_{\mu}^{T} \Gamma^{\mu} .
$$

The leading singularity of the first term on the right hand side of eq. (5.8) is determined by the d'Alembertian, i.e.

$$
-2 i \operatorname{Tr}\left[f^{\mu} \overrightarrow{\partial_{\mu}} \frac{1}{\square} \overleftarrow{\partial}_{\nu} f^{\nu} d_{0}^{-1}\right]
$$

In expression (7.10) it is described by the term involving the two-point function $B_{\mu \nu}\left(p_{1} ; 0, M\right)$. Accordingly, the first two terms of the low-energy expansion of this two-point function are relevant at order $p^{4}$. In this expansion the term of order 1 describes the contribution from the leading singularity while the term of order $p^{2}$ contributes to the next-to-leading one. The contributions from the heat coefficient $H_{1}$, occurring in eqs. (6.22), are readily identified as well. They are given in expression (7.11) by the term involving the quantity $\sigma$.

In this way one recovers the same result as was obtained by using the heat-kernel method. However, the evaluation of the term $-\frac{i}{2} \operatorname{Tr}\left(\delta D^{-1} \delta^{T} d_{0}^{-1}\right)$ by means of loop-integrals is painstaking, since due to the formal inversion of the operator $D$ in eq. ( 7.7$)$ the covariant form of the final result is not obvious.

Finally, we should mention that all three- and four-point functions with two or more massless propagators have infrared singularities in 4 dimensions. They would have to be taken care of, if we were to extract the contributions of order $p^{6}$ from the loop integrals, using the next terms in the low energy 
expansion of these functions. For instance, the next-to-leading order contribution of $C_{\mu \nu}\left(p_{1}, p_{2} ; 0,0, M\right)$ would enter at this order. The analysis shown in the appendix reveals that the low momentum expansion of this function is of the form

$$
C_{\mu \nu}\left(p_{1}, p_{2} ; 0,0, M\right)=P_{\mu \nu}\left(p_{1}, p_{2}\right)+Q_{\mu \nu}\left(p_{1}, p_{2}\right) \ln \left(\frac{-p_{1}^{2}}{M^{2}}\right)+\text { higher order },
$$

where $P_{\mu \nu}$ and $Q_{\mu \nu}$ are polynomials of second degree in $p_{1}$ and $p_{2}$. Only $P_{\mu \nu}$ contains a term of order 1 , which leads to a contribution of order $p^{4}$ through eq. (7.11). However, terms involving logarithms, like the one in eq. (7.15), do not contribute to the effective Lagrangian.

Note that we already encountered such terms in our calculation. They were hidden in the nonlocal determinant $\ln \operatorname{det} D$, which cancelled in the comparison of the generating functionals of the linear sigma model and of the effective theory in eq. (4.2). A similar situation would occur if we were to determine the contributions at order $p^{6}$. Note that in this case one has to calculate the generating functional in the linear sigma model as well as in the effective theory up to the two-loop level. Therefore additional terms would occur beyond lndet $\tilde{D}$, which represents the one loop graphs only. In this way one gets nonlocal terms in the linear sigma model and in the effective theory from loops, where a Goldstone boson propagates over long distances, leading to the above mentioned infrared problems. These nonlocal terms would, however, cancel in the matching of the generating functionals and the remaining local terms would lead to the coefficients $d_{i}$ at order $p^{4}$ and to analogous coefficients at order $p^{6}$.

The short distance expansion is only appropriate to get these local terms. If we use, for instance, a representation of the propagator $\left\langle x\left|D^{-1}\right| y\right\rangle$ by the heat-kernel as in eq. (6.16), it only makes sense to expand the heat-kernel for small values of the parameter $\lambda$, which corresponds to small distances $x-y$. Infrared singularities on the other hand show up at large $\lambda$ where the expansion does not make sense, especially if massless particles are present.

\section{Renormalization}

In order to obtain finite results for physical quantities one has to renormalize the bare constants $g, m$ and $h$ before the regulator can be removed (there is 
no wave-function renormalization at the order considered here, such that the external field $f^{A}$ does not require renormalization). This will indeed render the linear $O(N)$ sigma model finite, because it is a renormalizable theory. To analyze the ultraviolet behaviour of the determinant of the differential operator $\tilde{D}$ given in eq. (2.12) we again employ the heat kernel method. In Euclidean space-time the $d$-dimensional determinant may be defined as

$$
\begin{aligned}
\operatorname{lndet} \tilde{D} & =-\int_{0}^{\infty} \frac{\mathrm{d} \lambda}{\lambda} \operatorname{Tr} e^{-\lambda \tilde{D}} \\
& =-(4 \pi)^{-d / 2} \int_{0}^{\infty} \mathrm{d} \lambda \lambda^{-1-d / 2} \int \mathrm{d}^{d} x \operatorname{tr} \tilde{H}(x|\lambda| x),
\end{aligned}
$$

where the $\tilde{H}$ is the heat kernel of the operator $\tilde{D}$ defined in eq. (6.3). In this representation the divergences which are related to the ultraviolet behaviour show up at the lower end of the integration over $\lambda$. For $d=0,2,4, \ldots$ the determinant has poles. In order to identify the residues of these poles, we split the integration over $\lambda$ into an integral from 0 to $\lambda_{0}$ and a remainder. Using the Taylor series expansion (6.6) we obtain

$$
\begin{aligned}
\frac{1}{2} \operatorname{lndet} \tilde{D}= & \frac{1}{d} \int \mathrm{d}^{d} x \operatorname{tr} \tilde{H}_{0}(x \mid x)+\frac{1}{d-2} \frac{1}{4 \pi} \int \mathrm{d}^{d} x \operatorname{tr} \tilde{H}_{1}(x \mid x) \\
& +\frac{1}{d-4} \frac{1}{16 \pi^{2}} \int \mathrm{d}^{d} x \operatorname{tr} \tilde{H}_{2}(x \mid x)+\ldots
\end{aligned}
$$

With the result given in eq. (6.11), the the pole-term at $d=4$ turns out to be given by

$$
\frac{1}{d-4} \frac{1}{16 \pi^{2}} \int \mathrm{d}^{d} x\left\{\frac{1}{12} \operatorname{tr}\left(F_{\mu \nu} F_{\mu \nu}\right)+\frac{1}{2} \operatorname{tr}\left(\tilde{\sigma}^{2}\right)\right\} .
$$

Note that the differential operator $\tilde{D}$ is positive in the $O(N)$ symmetric phase of the linear sigma model. In this case the integrand in eq. (8.1) is exponentially damped as $\lambda$ goes to infinity, which expresses the fact that there are no infrared divergences in this phase. Since the divergence structure of a renormalizable field theory is the same in both, the symmetric and the spontaneously broken phase, the pole is removed by the following renormalization prescriptions:

$$
g=g_{r}\left(1-2(N+8) g_{r}\left(\hat{\lambda}_{0}\left(2 m^{2}\right)+\delta g\right)\right)+\mathcal{O}\left(g_{r}^{3}\right)
$$




$$
\begin{aligned}
m^{2} & =m_{r}^{2}\left(1-2(N+2) g_{r}\left(\hat{\lambda}_{0}\left(2 m^{2}\right)+\delta m^{2}\right)\right)+\mathcal{O}\left(g_{r}^{2}\right) \\
h & =h_{r}+\frac{1}{12} \hat{\lambda}_{0}\left(2 m^{2}\right)+\mathcal{O}\left(g_{r}\right) .
\end{aligned}
$$

These relations introduce the five finite but otherwise arbitrary constants $m_{r}, g_{r}, \delta m^{2}, \delta g$ and $h_{r}$. In the case of a (massive) Yang Mills theory, where the gauge field $F_{\mu}$ is a dynamical field, $h$ represents the inverse square of the gauge coupling constant. In the present context, where $F_{\mu}$ is an external field, loops involving gauge fields do not occur. The renormalization of $h$ encountered here only accounts for the renormalization of the gauge coupling constant which are due to loops of the field $\phi^{A}$. The finite renormalization constants $\delta m^{2}$ and $\delta g$ are in general eliminated by requiring two renormalization conditions. In addition to that, we will also replace the remaining two independent parameters $m_{r}$ and $g_{r}$ by two other quantities, the physical mass $M$ of the heavy particle and the low energy constant $F$, whose physical meaning will be discussed at the end of this section.

For the rest of this section we will use Minkowski space notation. The mass $M$ is determined by the pole position of the connected two-point function

$$
\left.-i \frac{\delta^{2}}{\delta f^{0}(x) \delta f^{0}(y)} W_{\sigma}\left[F_{\mu}, f\right]\right]\left.\right|_{F_{\mu}=f=0}=\left\langle 0\left|T\left(\phi^{0}(x) \phi^{0}(y)\right)\right| 0\right\rangle_{\text {conn }} .
$$

Note that we have embedded the unbroken symmetry group $O(N-1)$ in such a way that it does not act on the first component $\phi^{0}$ of the $N$-component field $\phi^{A}$. Thus, $\phi^{0}$ describes the massive mode while the remaining $N-1$ components $\phi^{i}, i=1, \ldots, N-1$, represent the Goldstone bosons. In order to evaluate the pole position, we use eqs. (2.11) and (2.36) as well as the solution $R$ of the equations of motion (2.16),

$$
\begin{aligned}
& R(x)-1=\int \mathrm{d}^{d} y G_{M}(x-y) \chi_{0}^{0}(y) \\
& \quad-3 m^{2} \int \mathrm{d}^{d} y \mathrm{~d}^{d} v \mathrm{~d}^{d} w G_{M}(x-y) G_{M}(y-v) G_{M}(y-w) \chi_{0}^{0}(v) \chi_{0}^{0}(w)+\ldots
\end{aligned}
$$

One obtains the following result for the pole position:

$$
\begin{aligned}
& M^{2}-i M \Gamma=2 m_{r}^{2}[ 1-2(N+2) g_{r} \delta m^{2}-\frac{1}{16 \pi^{2}} g_{r}\left\{9\left(1-\frac{\pi}{\sqrt{3}}\right)\right. \\
&+(N-1)(1+i \pi)\}],
\end{aligned}
$$


where $M$ and $\Gamma$ are the mass and width of the heavy particle. In terms of the renormalized quantities $m_{r}$ and $g_{r}$, the low-energy constant $F$ turns out to be

$$
F^{2}=\left(\frac{m_{r}^{2}}{g_{r}}\right)\left[1+2 g_{r}\left(\frac{1}{32 \pi^{2}}-(N+2) \delta m^{2}+(N+8) \delta g\right)\right] .
$$

Thus, one can eliminate the two renormalization constants $\delta m^{2}$ and $\delta g$ and replace the two parameters $m_{r}$ and $g_{r}$ by the physical mass $M$ and the lowenergy constant $F$ by requiring

$$
\begin{aligned}
M^{2} & =2 m_{r}^{2}, \\
F^{2} & =\frac{m_{r}^{2}}{g_{r}} .
\end{aligned}
$$

Thus, $\delta m^{2}$ and $\delta g$ are given by

$$
\begin{aligned}
(N+2) \delta m^{2} & =-\frac{1}{32 \pi^{2}}\left(9\left(1-\frac{\pi}{\sqrt{3}}\right)+N-1\right), \\
(N+8) \delta g & =(N+2) \delta m^{2}-\frac{1}{32 \pi^{2}} .
\end{aligned}
$$

The low-energy constants as well as the field $\chi$ turn out to be

$$
\begin{aligned}
\chi & =\frac{1}{F}\left(1-\frac{M^{2}}{F^{2}} \frac{1}{64 \pi^{2}}\right) f \\
d_{1} & =\frac{3 N-10}{6} \lambda_{0}+\frac{F^{2}}{2 M^{2}}-\frac{1}{192 \pi^{2}}\left(\frac{116+9 N-27 \sqrt{3} \pi}{3}-(3 N-10) \ln \frac{M^{2}}{\mu^{2}}\right) \\
d_{2} & =\frac{2}{3} \lambda_{0}-\frac{1}{96 \pi^{2}}\left(\frac{11}{3}-2 \ln \frac{M^{2}}{\mu^{2}}\right) \\
d_{3} & =\frac{N-1}{2} \lambda_{0}+\frac{F^{2}}{2 M^{2}}-\frac{1}{64 \pi^{2}}\left(\frac{49+3 N-9 \sqrt{3} \pi}{3}-(N-1) \ln \frac{M^{2}}{\mu^{2}}\right) \\
d_{4} & =(N-2) \lambda_{0}+\frac{F^{2}}{M^{2}}-\frac{1}{32 \pi^{2}}\left(15+N-3 \sqrt{3} \pi-(N-2) \ln \frac{M^{2}}{\mu^{2}}\right) \\
d_{5} & =-\frac{1}{6} \lambda_{0}-\frac{1}{192 \pi^{2}}\left(\frac{1}{6}+\ln \frac{M^{2}}{\mu^{2}}\right)
\end{aligned}
$$




$$
\begin{aligned}
& d_{6}=-\frac{1}{3} \lambda_{0}+\frac{1}{96 \pi^{2}}\left(\frac{11}{6}-\ln \frac{M^{2}}{\mu^{2}}\right) \\
& d_{7}=\frac{1}{192 \pi^{2}} \\
& d_{8}=\frac{1}{12} \lambda_{0}+h_{r}+\frac{1}{384 \pi^{2}} \ln \frac{M^{2}}{\mu^{2}} .
\end{aligned}
$$

Note that the low-energy constants are of the form

$$
d_{i}=\delta_{i} \lambda_{0}+\bar{d}_{i}
$$

where the $\bar{d}_{i}$ are finite and expressed in terms of physical quantities. It is furthermore important to note that the matching relation (4.1) between the linear sigma model and the effective theory determines both contributions to the low-energy constants, the finite parts $\bar{d}_{i}$ as well as the poles in $d=$ 4. These pole terms, $\delta_{i} \lambda_{0}$, occur in tree-diagrams of order $p^{4}$ and exactly cancel the corresponding poles in the contributions from loops with vertices from $\mathcal{L}_{2}$, as represented by the determinant in eq. (3.4). Since the effective theory describes the low-energy structure of the linear sigma model, the renormalization of the latter must indeed render the former finite as well.

Let us now compare the results for the low-energy constants given in eqs. (5.18) and (8.15) with those of refs. 9, 2, 30. Our results in eq. (5.18), where the low-energy constants are expressed in terms of bare quantities, agree with those in eq. (B.11) of ref. [9]. However, the renormalization condition used in that article differs from the one given in eq. (8.12) above, which has to be taken into account if the results of our eq. (8.15) are compared with eq. (B.12) of ref. [9].

The authors of ref. [2] integrate only over the heavy scalar field in the linear sigma model and, thus, obtain the functional $\Gamma_{\text {eff }}$ as defined in eq. (4.5) above. The expansion of this functional in terms of local quantities defines another set of parameters, $d_{i}^{\Gamma}$, which can be inferred from eqs. (5.1) and (5.5). We obtain, for example, $d_{2}^{\Gamma}=d_{5}^{\Gamma}=d_{6}^{\Gamma}=0$, which agrees with the results of ref. [2]. The constants $d_{2}, d_{5}$ and $d_{6}$, on the other hand, receive contributions from mixed loops and are different from zero. The effective Lagrangian,

\footnotetext{
${ }^{2}$ The low-energy constants $d_{i}$ are related to those in ref. [9] by $l_{3}=d_{3}-d_{4}, h_{1}=$ $d_{4}+d_{7}, h_{2}=d_{8}$, and $l_{i}=d_{i}$ for $i=1,2,4,5,6$, and to those in ref. [2] by $4 L_{1}=d_{1}, 4 L_{2}=$ $d_{2},-2 L_{9}=d_{6}$, and $L_{10}=d_{5}$.
} 
however, which describes the low-energy structure of the linear sigma model involves the low-energy constants $d_{i}$ as given in eqs. (5.18) and (8.15) and not the parameters $d_{i}^{\Gamma}$.

The analysis of the low-energy constants in ref. [3] includes the effects of dynamical gauge fields as well. In that article, the contributions from mixed loops are taken into account and, furthermore, it was found that loops containing gauge fields do not contribute to the low-energy constants at order $p^{4}$. For a more detailed comparison between the low-energy constants obtained in refs. [9] and [3] the reader is referred to ref. [3].

Let us conclude this section with two remarks about the low-energy constant $F$. At the order we are considering here it is identical with the constant $F_{\pi}$ which describes the coupling of the conserved currents to the Goldstone bosons. Recall that the gauge fields $F_{\mu}^{\alpha}$ couple to the conserved currents $J_{\mu}^{\alpha}$ given in eq. (2.6). By choosing a suitable basis for the $N-1$ Goldstone boson states $\left|\pi^{k}\right\rangle$ Lorentz invariance implies that these currents have the following matrix elements

$$
\left\langle 0\left|J_{\mu}^{\alpha}(0)\right| \pi^{k}(p)\right\rangle=i \delta^{\alpha k} F_{\pi} p_{\mu},
$$

which defines the coupling constant $F_{\pi}$. Note that only the $N-1$ currents which correspond to broken generators of the $O(N)$ symmetry couple to the Goldstone bosons. According to definition (8.17), the residue of the Goldstone boson pole of the two-point function

$$
-\left.i \frac{\delta^{2}}{\delta F_{\mu}^{\alpha}(x) \delta F_{\nu}^{\beta}(y)} W_{\sigma}\left[F_{\mu}, f\right]\right|_{F_{\mu}=f=0}=\left\langle 0\left|T\left(J_{\mu}^{\alpha}(x) J_{\nu}^{\beta}(y)\right)\right| 0\right\rangle_{\text {conn }}+\text { contact terms }
$$

is given by $F_{\pi}^{2}$. Note that the contact terms do not contribute to the pole position. Since $F_{\pi}$ is a low-energy constant, it may as well be determined from our representation of the generating functional $W_{\text {eff }}$. The explicit calculation yields

$$
F=F_{\pi}
$$

At tree-level, the coupling constant $F_{\pi}$ is identical with the vacuum expectation value $v$ of the field $\phi^{0}$, which is given by

$$
v \doteq\left\langle 0\left|\phi^{0}(x)\right| 0\right\rangle_{\text {conn }}=\left.\frac{\delta}{\delta f^{0}(x)} W_{\sigma}\left[F_{\mu}, f\right]\right|_{F_{\mu}=f=0} .
$$


Beyond tree-level, however, both quantities differ by corrections of order $g$ and the relation is given by

$$
v=F\left(1-\frac{M^{2}}{F^{2}} \frac{1}{64 \pi^{2}}\right) .
$$

This reflects the fact that the constant $F_{\pi}$ is not renormalized, because the normalization of the currents is fixed by the commutation relations. The vacuum expectation value $v$, on the other hand, depends on the normalization of the fields and, hence, on a convention.

Finally we want to make a comment on the validity of our first-order perturbative calculation. The physics of the linear $O(N)$ sigma model in the spontaneously broken phase can adequately be described by an effective theory, which contains only the Goldstone bosons, if energy and momenta are small compared to the mass of the heavy particle, i.e. if $p^{2} / M^{2}<<1$. The perturbative expansion in the coupling constant $g$, on the other hand, breaks down if this mass is too large, since $g \sim M^{2}$ according to eq. (8.12). As an example we consider the Standard Model values $N=4$ and $v=246 \mathrm{GeV}$. In this case perturbation theory in the parameter $g$ can no longer be applied if the mass is of the order of $1 \mathrm{TeV}$.

\section{Summary}

In this work we have analyzed the low-energy structure of the linear $O(N)$ sigma model from an effective field theory point of view. In the spontaneously

broken phase the spectrum of this theory consists of one massive mode and $N-1$ Goldstone bosons. At low energies, physical phenomena are dominated by the light particles of a theory, while the presence of heavier species manifests itself rather indirectly. In the low energy region, the nontrivial structure of the linear sigma model is related to the singularities of Green's functions which are associated with the Goldstone bosons. The effects of the heavy particle, on the other hand, admit a Taylor expansion and show up as simple power-like contributions. Hence, the low energy structure of this theory can adequately be described by an effective theory which contains only the Goldstone bosons. In the Lagrangian which describes the effective theory, effects of the heavy particle manifest themselves as an infinite tower 
of nonrenormalizable interactions which are accompanied by an infinite series of dimensionful low energy (coupling) constants. The requirement that both, the effective and the full theory describe the same low energy physics in principle determines the relationships between all these low energy constants and the parameters of the underlying theory. In more mathematical terms, the equivalence between the effective theory and the full theory requires that corresponding Green's functions have the same low energy structure. This can be expressed in a compact way as

$$
W_{\sigma}\left[F_{\mu}, f\right]=W_{e f f}\left[F_{\mu}, \chi\right],
$$

where $W_{\sigma}\left[F_{\mu}, f\right]$ and $W_{e f f}\left[F_{\mu}, \chi\right]$ are the generating functionals of the linear sigma model and of the effective theory, respectively. They depend on a set of external vector fields $F_{\mu}$ and scalar fields $\chi$ or $f$, which are coupled to the matter fields. Derivatives of these functionals with respect to the vector fields $F_{\mu}$ generate Green's function of the $O(N)$ currents, while derivatives with respect to the scalar fields $\chi$ or $f$ generate Green's functions of the matter fields. Equation (9.1) should not be understood as an identity but rather as an asymptotic equality in the low energy region. It determines the low energy constants as well as the relation between the external scalar fields $\chi$ and $f$. More explicitly, eq. (9.1) requires the following relation between full path integrals

$$
\int \mathrm{d} \mu[U] e^{i \int \mathrm{d}^{d} \mathcal{L}_{e f f}\left(U, F_{\mu}, \chi\right)}=\int \mathrm{d} \mu[U] \mathrm{d} \mu[R] e^{i \int \mathrm{d}^{d} \mathcal{L}_{\sigma}\left(R, U, F_{\mu}, f\right)},
$$

where the effective Lagrangian $\mathcal{L}_{\text {eff }}\left(U, F_{\mu}, \chi\right)$ depends on the external sources and on the fields $U$ of the Goldstone bosons, while the Lagrangian of the linear sigma model $\mathcal{L}_{\sigma}\left(R, U, F_{\mu}, f\right)$ depends on the field $R$ of the heavy degree of freedom as well. At the classical level, this relation between integrals is equivalent to the following relation between integrands

$$
e^{i \int \mathrm{d}^{d} \mathcal{L}_{e f f}\left(U, F_{\mu}, \chi\right)}=\int \mathrm{d} \mu[R] e^{i \int \mathrm{d}^{d} \mathcal{L}_{\sigma}\left(R, U, F_{\mu}, f\right)} .
$$

This equivalence, however, is lost if quantum corrections are taken into account. In other words, beyond tree-level, the correct matching condition is given by relation (9.2) between the full integrals. In this sense, integrating out heavy particles should rather be understood as replacing the full theory by an effective one which describes the same low energy physics. 
At low energies, Green's functions receive nonlocal contributions which are related to the propagation of light particles over large distances. Since both, the effective and the full theory have the same light particle content, all nonlocal contributions drop out of the matching relation. The remaining local terms involve the propagation of heavy particles over short distances. It has been shown in this work how the heat kernel technique can be used to evaluate the short-distance expansion of these terms and thus determine the functional relationships between the low energy constants and the parameters of the full theory. These relationships can, however, also be evaluated by means of loop integrals in momentum space. In order to present a detailed description of both methods we have explicitly evaluated the effective Lagrangian for the linear $O(N)$ sigma model in the spontaneously broken phase up to the order $p^{4}$. In contrast to the short distance expansion, the evaluation based on loop-integrals is not covariant during intermediate steps of the calculation. The momentum space picture is useful, however, to understand why eq. (9.3) does not define the correct effective Lagrangian beyond tree-level. The local terms in the matching condition receive quantum corrections from two types of Feynman diagrams, cf. eq. (4.3), those which involve propagators only of the heavy degrees of freedom and those which involve propagators of both, heavy and light particle species. The latter ones are obviously missed in eq. (9.3). Finally we have expressed the low energy constants in terms of the mass $M$ of the heavy particle and the decay constant $F$.

\section{Acknowledgements}

We are grateful to H. Leutwyler for many enlightening discussions and a critical reading of this manuscript. One of us (A.S.) is furthermore indebted to M. Dugan, H. Georgi and S. Vandermark for interesting discussions. He also wants to thank the members of the Harvard Physics Department for their kind hospitality.

\section{Appendix}

In this appendix we briefly want to discuss how the low energy expansion of $n$ -

point functions can be constructed. As a particular example we shall consider 
the two three-point functions $C_{\mu \nu}\left(p_{1}, p_{2} ; 0, M, M\right)$ and $C_{\mu \nu}\left(p_{1}, p_{2} ; 0,0, M\right)$. Note, that the latter one has infrared singularities while the former one is regular for small momenta. In Euclidean space-time one has

$$
\begin{aligned}
& C_{\mu \nu}\left(p_{1}, p_{2} ; 0, m_{2}, m_{3}\right) \\
& \quad=\int \frac{\mathrm{d}^{d} p}{(2 \pi)^{d}} \frac{p_{\mu} p_{\nu}}{p^{2}\left(\left(p+p_{1}\right)^{2}+m_{2}^{2}\right)\left(\left(p+p_{1}+p_{2}\right)^{2}+m_{3}^{2}\right)} .
\end{aligned}
$$

A convenient Feynman parametrization is given by

$$
\begin{array}{rr}
\alpha\left[(1-\beta)\left(\left(p+p_{1}\right)^{2}+m_{2}^{2}\right)+\beta\left(\left(p+p_{1}+p_{2}\right)^{2}+m_{3}^{2}\right)\right] & (\mathrm{A} .2) \\
+(1-\alpha) p^{2} & \doteq p^{2}+2 p k+\Delta,
\end{array}
$$

with

$$
\begin{aligned}
k & =\alpha\left(p_{1}+\beta p_{2}\right) \\
\Delta & =\alpha\left[(1-\beta)\left(m_{2}^{2}+p_{1}^{2}\right)+\beta\left(m_{3}^{2}+\left(p_{1}+p_{2}\right)^{2}\right)\right] .
\end{aligned}
$$

The integration over $p$ yields

$$
C_{\mu \nu}=\int_{0}^{1} \mathrm{~d} \alpha \alpha \int_{0}^{1} \mathrm{~d} \beta\left(k_{\mu} k_{\nu} F(0)+\frac{1}{2} \delta_{\mu \nu} F(1)\right),
$$

where

$$
F(l)=\frac{1}{(4 \pi)^{d / 2}} \frac{\Gamma\left(3-l-\frac{d}{2}\right)}{\left(\Delta-k^{2}\right)^{3-l-d / 2}} .
$$

In the following we will furthermore use dimensionless momenta

$$
q_{i} \doteq \frac{p_{i}}{M}
$$

In the case of $C_{\mu \nu}\left(p_{1}, p_{2} ; 0, M, M\right), m_{2}=m_{3}=M$ and we have

$$
\Delta-k^{2}=\alpha M^{2}\left[1+f_{1}\left(\alpha, \beta, q_{1}, q_{2}\right)\right],
$$

where the function

$$
f_{1}\left(\alpha, \beta, q_{1}, q_{2}\right) \doteq(1-\beta) q_{1}^{2}+\beta\left(q_{1}+q_{2}\right)^{2}-\alpha\left(q_{1}+\beta q_{2}\right)^{2}
$$


is of order $q^{2}$. Thus, the three-point function $C_{\mu \nu}\left(p_{1}, p_{2} ; 0, M, M\right)$ is regular for small momenta and the low energy expansion is obtained by expanding the integrand in powers of $f_{1}$. At leading order we get

$$
C_{\mu \nu}\left(p_{1}, p_{2} ; 0, M, M\right)=-\frac{1}{2} \delta_{\mu \nu}\left\{\hat{\lambda}_{0}\left(M^{2}\right)+\frac{1}{32 \pi^{2}} \frac{1}{2}\right\} .
$$

In the case of $C_{\mu \nu}\left(p_{1}, p_{2} ; 0,0, M\right), m_{2}=0 \& m_{3}=M$ and we get

$$
\Delta-k^{2}=\alpha M^{2} F\left(\alpha, \beta, q_{1}, q_{2}\right),
$$

where

$$
F\left(\alpha, \beta, q_{1}, q_{2}\right) \doteq \beta+(1-\beta) q_{1}^{2}+\beta\left(q_{1}+q_{2}\right)^{2}-\alpha\left(q_{1}+\beta q_{2}\right)^{2} .
$$

Since $F\left(\alpha, \beta, q_{1}, q_{2}\right)$ vanishes iff $\beta=0$ and $\alpha=1$, we encounter an endpoint singularity in this case. Therefore the three-point function $C_{\mu \nu}\left(p_{1}, p_{2} ; 0,0, M\right)$ will have a branch point at $q_{1}^{2}=0$. In order to analyze the behaviour of this function at small momenta, we separate the term linear in $\beta$ and $1-\alpha$ as follows

$$
F\left(\alpha, \beta, q_{1}, q_{2}\right)=\left(\beta\left(1+q_{2}^{2}\right)+(1-\alpha) q_{1}^{2}\right)\left(1+f_{2}\left(\alpha, \beta, q_{1}, q_{2}\right)\right),
$$

where the quantity

$$
f_{2}\left(\alpha, \beta, q_{1}, q_{2}\right) \doteq \frac{\beta}{\beta\left(1+q_{2}^{2}\right)+(1-\alpha) q_{1}^{2}}\left(-\beta \alpha q_{2}^{2}+2(1-\alpha) q_{1} q_{2}\right)
$$

is of order $q^{2}$ in the region of integration. Note, that this function is wellbehaved even in the limit $\beta \rightarrow 0^{+}$and $\alpha \rightarrow 1^{-}$. Thus we can expand this integrand in powers of $f_{2}$. In this way one obtains

$$
\begin{aligned}
& \int_{0}^{1} \mathrm{~d} \alpha \alpha \int_{0}^{1} \mathrm{~d} \beta k^{\mu} k^{\nu} F(0) \\
& \quad=\frac{1}{16 \pi^{2}}\left[q_{1}^{\mu} q_{1}^{\nu}\left(\frac{11}{18}-\frac{1}{3} \ln q_{1}^{2}\right)+\frac{1}{6} q_{2}^{\mu} q_{2}^{\nu}+\frac{1}{3}\left(q_{1}^{\mu} q_{2}^{\nu}+q_{1}^{\nu} q_{2}^{\mu}\right)\right]+\ldots
\end{aligned}
$$

and

$$
\begin{aligned}
& \int_{0}^{1} \mathrm{~d} \alpha \alpha \int_{0}^{1} \mathrm{~d} \beta F(1) \\
& \quad=-\hat{\lambda}_{0}\left(M^{2}\right)+\frac{1}{32 \pi^{2}}\left[\frac{1}{2}-\frac{11}{18} q_{1}^{2}-\frac{2}{3} q_{2}^{2}-\frac{2}{3} q_{1} q_{2}+\frac{1}{3} q_{1}^{2} \ln q_{1}^{2}\right]+\ldots
\end{aligned}
$$


For completeness sake we finally present all the terms in the low energy expansions of the $n$-point functions that we use in section 7 (in Minkowski metric):

$$
\begin{aligned}
B\left(p_{1} ; M, M\right)= & -2 \hat{\lambda}_{0}\left(M^{2}\right)-\frac{1}{16 \pi^{2}} \\
B\left(p_{1} ; 0, M\right)= & -2 \hat{\lambda}_{0}\left(M^{2}\right) \\
B^{\mu}\left(p_{1} ; 0, M\right)= & p_{1}^{\mu}\left(\hat{\lambda}_{0}\left(M^{2}\right)+\frac{1}{32 \pi^{2}} \frac{1}{2}\right) \\
B^{\mu \nu}\left(p_{1} ; 0, M\right)= & -\frac{M^{2}}{2} g^{\mu \nu}\left(\hat{\lambda}_{0}\left(M^{2}\right)-\frac{1}{32 \pi^{2}} \frac{1}{2}\right) \\
& +\frac{p_{1}^{2}}{6} g^{\mu \nu}\left(\hat{\lambda}_{0}\left(M^{2}\right)+\frac{1}{32 \pi^{2}} \frac{1}{6}\right) \\
& -\frac{2}{3} p_{1}^{\mu} p_{1}^{\nu}\left(\hat{\lambda}_{0}\left(M^{2}\right)+\frac{1}{32 \pi^{2}} \frac{2}{3}\right) \\
C^{\mu \nu}\left(p_{1}, p_{2} ; 0, M, M\right)= & \frac{1}{2} g^{\mu \nu}\left(\hat{\lambda}_{0}\left(M^{2}\right)+\frac{1}{32 \pi^{2}} \frac{1}{2}\right) \\
C^{\mu \nu}\left(p_{1}, p_{2} ; 0,0, M\right)= & \frac{1}{2} g^{\mu \nu}\left(\hat{\lambda}_{0}\left(M^{2}\right)-\frac{1}{32 \pi^{2}} \frac{1}{2}\right) \\
C^{\mu \nu \rho}\left(p_{1}, p_{2} ; 0,0, M\right)= & -\frac{g^{\mu \nu} p_{1}^{\rho}}{3}\left(\hat{\lambda}_{0}\left(M^{2}\right)-\frac{1}{32 \pi^{2}} \frac{1}{3}\right) \\
& -\frac{g^{\mu \nu} p_{2}^{\rho}}{6}\left(\hat{\lambda}_{0}\left(M^{2}\right)+\frac{1}{32 \pi^{2}} \frac{1}{6}\right) \\
& -\operatorname{permutations~of~}(\mu \nu \rho) \\
D^{\mu \nu \rho \sigma}\left(p_{1}, p_{2}, p_{3} ; 0, M, 0, M\right)= & -\frac{1}{12}\left(g^{\mu \nu} g^{\rho \sigma}+g^{\mu \rho} g^{\nu \sigma}+g^{\mu \sigma} g^{\nu \rho}\right) \\
& \left(\hat{\lambda}_{0}\left(M^{2}\right)+\frac{1}{32 \pi^{2}} \frac{1}{6}\right) \\
D^{\mu \nu \rho \sigma}\left(p_{1}, p_{2}, p_{3} ; 0,0,0, M\right)= & -\frac{1}{12}\left(g^{\mu \nu} g^{\rho \sigma}+g^{\mu \rho} g^{\nu \sigma}+g^{\mu \sigma} g^{\nu \rho}\right) \\
& \left(\hat{\lambda}_{0}\left(M^{2}\right)-\frac{1}{32 \pi^{2}} \frac{5}{6}\right)
\end{aligned}
$$




\section{References}

[1] T. Appelquist and C. Bernard, Phys. Rev. D22 (1980) 200

A. C. Longhitano, Phys. Rev. D22 (1980) 1166

T. Appelquist in "Gauge Theories and Experiments at High Energies" (K. C. Bowler and D. G. Sutherland, Eds.), Scottish University Summer School in Physics, St. Andrews, 1980

A. C. Longhitano, Nucl. Phys. B188 (1981) 118.

[2] D. Espriu, in "Proceedings of the workshop on Effective Field Theories of the Standard Model" (Ulf-G. Meißner, Ed.), (Dobogókö 1991), World Scientific, Singapore, 1992

D. Espriu And M. J. Herrero, Nucl. Phys. B373 (1992) 117.

[3] M. J. Herrero and E. R. Morales, Nucl. Phys. B418 (1994) 431 M. J. Herrero and E. R. Morales, "The electroweak chiral parameters for a heavy Higgs in the Standard Model", Madrid, Autonoma University, FTUAM 94/12 May 1994.

[4] D. Espriu and J. Matias, "Some Remarks on the Matching Conditions", Barcelona University, UB-ECM-PF-94/12 June 1994.

[5] S. Weinberg, Phys. Rev. D19 (1979) 1277

L. Susskind, Phys. Rev. D20 (1979) 2619

E. Farhi And L. Susskind, Phys. Rep. 74 (1981) 277.

[6] For recent reviews on the use of effective Lagrangians in electroweak physics, see

F. Feruglio, Int. J. Mod. Phys. A8 (1993) 4937

J. Wudka, Int. J. Mod. Phys. A9 (1994) 2301.

[7] H. Georgi, Annu. Rev. Nucl. Part. Sci. 43 (1993) 209.

[8] B. Ovrut and H. J. Schnitzer, Phys. Rev. D24 (1981) 1695.

[9] J. Gasser and H. Leutwyler, Ann. Phys. 158 (1984) 142. 
[10] S. Coleman, J. Wess and B. Zumino, Phys. Rev. 177 (1969) 2239 C. G. Callan, S. Coleman, J. Wess and B. Zumino, Phys. Rev. 177 (1969) 2247.

[11] H. LeutwyleR, "On the foundations of chiral perturbation theory", Bern University Preprint, BUTP-93/24 Aug. 1993, Ann. Phys., in print.

[12] S. Weinberg, Physica A 96 (1979) 327.

[13] J. Schwinger, Phys. Rev. 82 (1951) 664

P. B. Gilkey, "The Index Theorem and the Heat Equation", Publish or Perish, Boston, 1974

P. B. Gilkey, "Invariance Theory, The Heat Equation, And the Atiyah-Singer Index Theorem", Publish or Perish, Wilmington, 1984. 